\title{
Minimizing normalized volumes of valuations
}

\author{
Chi Li
}

July 19, 2017

\begin{abstract}
For any $\mathbb{Q}$-Gorenstein klt singularity $(X, o)$, we introduce a normalized volume function $\widehat{v o l}$ that is defined on the space of real valuations centered at $o$ and consider the problem of minimizing vol. We prove that the normalized volume has a uniform positive lower bound by proving an Izumi type estimate for any $\mathbb{Q}$-Gorenstein klt singularity. Furthermore, by proving a properness estimate, we show that the set of real valuations with uniformly bounded normalized volumes is compact, and hence reduce the existence of minimizers for the normalized volume functional vol to a conjectural lower semicontinuity property. We calculate candidate minimizers in several examples to show that this is an interesting and nontrivial problem. In particular, by using an inequality of de-Fernex-Ein-Mustaţă, we show that the divisorial valuation associated to the exceptional divisor of the standard blow up is a minimizer of vol for a smooth point. Finally the relation to Fujita's work on divisorial stability is also pointed out.
\end{abstract}

\section{Contents}

1 Introduction and main results 2

2 Preliminaries $\quad 3$

2.1 Volumes and $\log$ discrepancies of valuations . . . . . . . . . . . . 3

2.2 Weights vs. $\log$ discrepancies . . . . . . . . . . . . . . 5

$\begin{array}{llr}3 & \text { Izumi type estimate } & 6\end{array}$

4 Minimizing normalized volume $\quad 9$

4.1 Proof of Theorem 4.1 . . . . . . . . . . . . . . . 11

5 Examples $\quad 13$

6 Relation to Fujita's divisorial stability 17

7 Questions and discussions $\quad 20$

7.1 Some conjectures . . . . . . . . . . . . . . . . . 20

8 Appendix I: Candidate minimizer for D-type and E-type singularities 21

$9 \quad$ Appendix II: Second proof of Theorem 3.2

10 Acknowledgment 


\section{Introduction and main results}

This paper is motivated by the following question:

Given a $\mathbb{Q}$-Gorenstein klt singularity $(X, o)$, is there an optimal way of rescaling it to obtain an affine cone that has a canonical metric structure?

This will be made more precise later, and here we just give some rough explanation. A rescaling in the above question will be represented by a real valuation centered at the singularity $o \in X$ and the affine cone is given by the spectrum of the associated graded ring (assuming that the latter is finitely generated). To define the "optimal way", we first introduce the normalized volume function that is defined on $\mathrm{Val}_{X, o}$, the space of real valuations that are centered at $o \in X$ :

$$
\widehat{\operatorname{vol}}(v)=A(v)^{n} \operatorname{vol}(v) \text {, for any } v \in \operatorname{Val}_{X, o} .
$$

Here $A_{X}(v)$ denotes the log-discrepancy of $v$ and $\operatorname{vol}(v)$ denotes the volume of $v$. We refer to Section 2.1 for their definitions. Then we seek for the minimizer of $\widehat{\operatorname{vol}}(v)$ among all real valuations that are centered at $o$.

This minimization question is motivated by the recent study of Kähler-Einstein metrics (we will refer to section ?? for some discussion of the study that motivates our problem). The transition to the current purely algebro-geometric question is however inspired by a corresponding volume minimization phenomenon in Sasaki-Einstein geometry that was discovered by Martelli-Sparks-Yau in [MSY08] (see also [CS12]), which in some sense is a special case of the general procedure discussed here (cf. Section 2.2 and [LL16]). A key observation that leads to the much more general setting is that a normalization involving weights of holomorphic volume forms in Sasaki-Einstein geometry can be equivalently rephrased by using the log discrepancy of valuations (see Section $2.2)$.

In this paper we shall introduce the basic set-up of this minimization problem, illustrate it by motivating examples and prove estimates that will be useful for further developments.

A basic estimate we prove is stated in the following theorem.

Theorem 1.1. Assume $(X, o)$ is a $\mathbb{Q}$-Gorenstein klt singularity. There exists a positive constant $K=K(X, o)>0$ such that for any valuation $v$ centered at $o \in X$ with $A_{X}(v)<+\infty$, the following inequality holds:

$$
A_{X}(v)^{n} \cdot \operatorname{vol}(v) \geq K \frac{A_{X}(v)}{v(\mathfrak{m})}
$$

where $\mathfrak{m}$ is the maximal ideal defining $o \in X$.

The infimum of the left-hand-side in (16) is a new invariant of the klt singularity and seems interesting to be studied further. To prove this estimate, there are two main ingredients that are of independent interests. The first is an Izumi-type estimate which generalizes a well-known estimate for smooth points:

Theorem 1.2 (=Theorem 3.1). Let $(X, o)$ be a $\mathbb{Q}$-Gorenstein klt singularity. Then there exists a constant $c_{1}=c_{1}(X, o)>0$ such that

$$
v(\mathfrak{m}) \operatorname{ord}_{o} \leq v \leq c_{1} \cdot A_{X}(v) \operatorname{ord}_{o},
$$

for any valuation $v$ centered at 0 . As a consequence, there is a uniform lower bound $\widehat{\operatorname{vol}}(v) \geq \mathfrak{e}(\mathfrak{m}) / c_{1}^{n}>0$ for any $v \in \operatorname{Val}_{X, o}$, where $e(\mathfrak{m})$ is the Hilbert-Samuel multiplicity of $\mathfrak{m}$.

The other ingredient is the following more technical estimate, which is related to a volume formula of Favre-Jonsson for $v \in \mathrm{Val}_{\mathbb{C}^{2}, 0}$. 
Theorem 1.3 (=Theorem 4.1). Assume $(X, o)$ is a $\mathbb{Q}$-Gorenstein klt singularity. There exists a positive constant $c_{2}=c_{2}(X, o)>0$ such that for any valuation centered at $o$, the following inequality holds:

$$
\operatorname{vol}(v) \geq c_{2}\left(\sup _{\mathfrak{m}} \frac{v}{\operatorname{ord}_{o}}\right)^{1-n} \frac{1}{v(\mathfrak{m})}
$$

The estimate in Theorem 1.1 strongly suggests that the minimizer should exist. In fact, Theorem 1.1 reduces the existence to the lower semicontinuity of $\widehat{\mathrm{vol}}$ on $\mathrm{Val}_{X, o}$ (see Corollary 4.4). We make the following

Conjecture 1.4. For any $\mathbb{Q}$-Gorenstein klt singularity $(X, o)$, there exists a unique minimizing valuation of $\widehat{\mathrm{vol}}$ on $\mathrm{Val}_{X, o}$.

We point out that the Conjecture 1.4 is non-trivial even for the smooth case. By using an inequality of de-Fernex-Ein-Mustață, we will prove in Proposition 5.2 that the exceptional divisor of the standard blow up is a minimizer. In a following paper [Li15b], we will study the case when $X$ is an affine cone over a $\mathrm{K}$-semistable $\mathbb{Q}$-Fano variety.

Finally by deriving the volume formula for specific divisorial valuations, we point out that there is a close relation of our minimization problem to the work of Fujita on divisorial semi-stability.

We end this introduction by outlining the organization of the paper. In the next section, we recall the definition of volumes and $\log$ discrepancies for valuations and explain a key observation on Sasaki-Einstein geometry that leads to our formulation of the problem. In section 3, we prove the Izumi type estimate stated in Theorem 1.2. In section 4, we prove Theorem 1.1 by proving the technical estimate in Theorem 4.1. In Section 5, we discuss several examples including the case of smooth point. In section 6 , we point out the relation between the minimizations of normalized volumes and Fujita's work. In section 7.1 we propose several conjectures that are natural from our point of view. In Appendix I, we write down the candidate minimizer for $D$-type and $E$-type singularities. In Appendix II, following the suggestion of a referee, we give an alternative proof of Theorem 3.2 by using the argument of Boucksom-Favre-Jonsson.

\section{Preliminaries}

\subsection{Volumes and log discrepancies of valuations}

Let $X^{n}$ be an $n$-dimensional normal affine variety. Fix a closed point $o \in X$ and let $R:=\mathcal{O}_{X, o}$ be the local ring of $X$ at $o$ with the maximal ideal denoted by $\mathfrak{m}$. Let $\mathrm{Val}_{X, o}$ denote the space of all real valuations on $\mathbb{C}(X)$ with center $o$ on $X$. For any $v \in \operatorname{Val}_{X, o}$, we define the volume of $v$ following Ein-Lazarsfeld-Smith (see [ELS03]):

$$
\operatorname{vol}(v)=\limsup _{r \rightarrow+\infty} \frac{\operatorname{length}_{R}\left(R / \mathfrak{a}_{r}\right)}{r^{n} / n !},
$$

where $\mathfrak{a}_{r}=\{f \in R ; v(f) \geq r\}$. By [ELS03, Mus02, Cut12], we know that:

$$
\operatorname{vol}(v)=e\left(\mathfrak{a}_{\bullet}\right):=\lim _{r \rightarrow+\infty} \frac{e\left(\mathfrak{a}_{r}\right)}{r^{n}} .
$$

Here the Hilbert-Samuel multiplicity $e\left(\mathfrak{a}_{r}\right)$ is defined as follows:

$$
e\left(\mathfrak{a}_{r}\right)=\lim _{p \rightarrow+\infty} \frac{\operatorname{length}\left(R / \mathfrak{a}_{r}^{p}\right)}{p^{n} / n !} .
$$

From now on, we will restrict our attention to the class of $\mathbb{Q}$-Gorenstein klt singularities. Recall the following standard definitions:

Definition 2.1. $\quad$ 1. $(X, o)$ is $\mathbb{Q}$-Gorenstein if there exists an integer $m \in \mathbb{Z}$ such that $m K_{X}$ is a Cartier divisor near $o \in X$. 
2. Let $(X, o)$ be a $\mathbb{Q}$-Gorenstein singularity. $(X, o)$ is klt (Kawamata log terminal) if for any divisor $E$ over $(X, o)$, we have $A_{X}(E)>0$.

Following [JM10] and [BFFU13], we briefly recall the definition of log discrepancy function $A(v)=A_{X}(v)$ for any valuation centered at $o: v \in \operatorname{Val}_{X, o}$. If $v=\operatorname{ord}_{E}$ is a divisorial valuation for an exceptional divisor $E$ over $o$ such that there is a birational morphism $\pi: Y \rightarrow X$ and $E$ is a prime divisor on $Y$ that is contracted to $o \in X$, then we define its $\log$ discrepancy as the coefficient of $E$ in $K_{Y / X}+E$ :

$$
K_{Y}+E=\pi^{*} K_{X}+A(E) E+F,
$$

where $\operatorname{Supp}(F)$ does not contain $E$. In general, one first defines the log discrepancy for any quasi-monomial valuation as follows. Assume $\mu: Y \rightarrow X$ is a resolution of singularities. $X$ and $\underline{y}=\left(y_{1}, \ldots, y_{r}\right)$ is a system of algebraic coordinates at a point $y \in Y$. By [JM10, Proposition 3.1], to every $\alpha=\left(\alpha_{1}, \ldots, \alpha_{r}\right) \in \mathbf{R}_{\geq 0}^{n}$ one can associate a unique valuation $\operatorname{val}_{\alpha}=\operatorname{val}_{\underline{y}, \alpha} \in \mathrm{Val}_{X}$ with the following property: whenever $f \in \mathcal{O}_{Y, \eta}$ is written in $\widehat{\mathcal{O}_{Y, \eta}}$ as $f=\sum_{\beta \in \mathbb{Z}_{\geq 0}^{r}} c_{\beta} y^{\beta}$, with each $c_{\beta}$ either zero or unit, we have

$$
\operatorname{val}_{\alpha}(f)=\min \left\{\langle\alpha, \beta\rangle \mid c_{\beta} \neq 0\right\} .
$$

The set of all such valuations (called quasi-monomial valuations or equivalently Abhyankar valuations, see [ELS03]) is denoted by $\mathrm{QM}_{\eta}(Y, D)$. If $\eta$ is the generic point of a connected component of intersection of $D_{1}, \ldots, D_{r}$ of $D$, then the map

$$
\mathrm{QM}_{\eta}(Y, D) \rightarrow \mathbb{R}^{r}, \quad v \mapsto\left(v\left(D_{1}\right), \ldots, v\left(D_{r}\right)\right)
$$

gives a homeomorphism onto the cone $\mathbb{R}_{\geq 0}^{r}$ (see [JM10, Lemma 4.5]). The log discrepancy function for such quasi-monomial valuation is defined as follows:

$$
A_{X}(v)=\sum_{i=1}^{r} v\left(D_{i}\right) \cdot A_{X}\left(\operatorname{ord}_{D_{i}}\right)=\sum_{i=1}^{r} v\left(D_{i}\right) \cdot\left(1+\operatorname{ord}_{D_{i}}\left(K_{Y / X}\right)\right) .
$$

In [JM10], Jonsson-Mustaţă defined $A(v)$ for any real valuation on $\mathbb{C}^{n}$. They first showed that there is a retraction map $r: \operatorname{Val}_{X} \rightarrow \mathrm{QM}(Y, D)$ such that it induces a homomeomorphism

$$
r: \operatorname{Val}_{X} \rightarrow \lim _{(\overleftarrow{Y, D})} \mathrm{QM}(Y, D)
$$

For any real valuation $v \in \operatorname{Val}_{X, o}$, one can then define:

$$
A(v)=A_{X}(v):=\sup _{(Y, D)} A\left(r_{Y, D}(v)\right)
$$

where the supremum is over all $\log$-smooth pairs $(Y, D)$ over $X$. Jonsson-Mustaţă's construction has been generalized to the singular case in [BFFU13] by following the same scheme of approximations.

Remark 2.2. In this paper we will only work with the class of $\mathbb{Q}$-Gorenstein singularities. Notice that the log discrepancy and klt condition can be defined for all normal singularities following the work of de-Fernex-Hacon [dFH09] (see also [BFF12, BFFU13]) and most discussions in this paper can indeed be generalized correspondingly (pointed out to me by a referee). However to avoid technical complications we leave the generalization to future works.

Notice that with the above definitions and notations, $A(\lambda v)=\lambda A(v)$ and $\operatorname{vol}(\lambda v)=$ $\lambda^{-n} \operatorname{vol}(v)$ for any $\lambda>0$ and $v \in \operatorname{Val}_{X, o}$. So the function $A_{X}(v)^{n} \operatorname{vol}(v)$ is a scaling invariant function on $\operatorname{Val}_{X, o}$. This function is well defined if $A_{X}(v)<+\infty$ (see Section $4)$. Where there is a torus action on $(X, o)$ with $o$ being the unique attracting point, the restriction of $\widehat{\text { vol }}$ to the space of toric invariant valuations appeared in Sasaki-Einstein geometry (in a disguised form) which we will discuss next. 


\subsection{Weights vs. log discrepancies}

In this section, we relate the weight of holomorphic volume form to the log discrepancy of torus invariant valuation. This is the key observation that leads to our general minimization problem.

Assume that $X=\operatorname{Spec}\left(\mathbb{C}\left[z_{1}, \ldots, z_{n}\right] / I(X)\right)$ is a normal affine variety over $\mathbb{C}$ with a $\left(\mathbb{C}^{*}\right)^{r}$-action. Assume that the action is free outside $o$ and $o$ is an attracting fixed point. We have the weight decomposition:

$$
R:=\mathbb{C}\left[z_{1}, \ldots, z_{n}\right] / I(X)=\bigoplus_{\alpha \in \Gamma^{*}} R_{\alpha} .
$$

We assume that $R_{\alpha} \neq 0$ for any $\alpha \in \Gamma^{*}$. The Reeb cone is a cone $\mathcal{C} \subset \mathfrak{t}$ consisting of all $\xi \in \mathfrak{t} \cong \mathbb{R}^{r}$ such that $\alpha(\xi)>0$ for all $\alpha \in \Gamma^{*}$. If $\xi \in \mathcal{C}$, then one can define the index character:

$$
f(t, \xi)=\sum_{\alpha \in \Gamma^{*}} e^{-t \alpha(\xi)} \operatorname{dim} R_{\alpha},
$$

which has an expansion ([MSY08], [CS12]) near $t=0$ :

$$
f(t, \xi)=\frac{a_{0}(\xi)}{t^{n}}+O\left(t^{1-n}\right) .
$$

It's easy to see that $a_{0}(\xi)$ is a homogeneous function of $\xi$ of homogeneous degree of $-n$ (see [MSY08]).

If $\xi$ is rational, then $e^{t \xi}$ generates a $\mathbb{C}^{*}$ action. $(E, \Delta)=X /\left\langle e^{t \xi}\right\rangle$ is an algebraic stack and there is an orbifold line bundle $L \rightarrow(E, \Delta)$ such that the underlying variety $X$ becomes an orbifold cone over $(E, \Delta)$. In other words we have:

$$
E=\operatorname{Proj} \bigoplus_{m=0}^{\infty} H^{0}(E, m L) \text { and } X=\operatorname{Spec} \bigoplus_{m=0}^{\infty} H^{0}(E, m L):=C(E, L)
$$

If $\xi=w \partial_{w}$ is the standard rescaling vector where $w$ is a linear coordinate along the fibre of $X \backslash\{o\} \rightarrow E$, then it's easy to verify that $a_{0}(\xi)=L^{n-1}$. On the other hand, any Reeb vector field $\xi$ determines a real valuation $\mathrm{wt}_{\xi} \in \mathrm{Val}_{X, o}$ in the following way:

$$
\operatorname{wt}_{\xi}(f)=\min _{\alpha \in \Gamma^{*}}\left\{\langle\alpha, \xi\rangle ; f_{\alpha} \neq 0\right\} \text { for any } f=\sum_{\alpha} f_{\alpha} \in R \text {. }
$$

Lemma 2.3. $\operatorname{vol}\left(\mathrm{wt}_{\xi}\right)=a_{0}(\xi)$ for $\xi=w \partial_{w}$.

Proof. Since both sides are homogeneous of degree $-n$ in $\xi$. We can normalize $\xi$ such that it corresponds to the standard scaling along the fibre of $L$. Then it's easy to see that

$$
\mathfrak{a}_{r}\left(\mathrm{wt}_{\xi}\right) / \mathfrak{a}_{r+1}\left(\mathrm{wt}_{\xi}\right) \cong H^{0}\left(X, L^{r}\right) .
$$

So we can calculate:

$$
\begin{aligned}
\operatorname{dim}_{\mathbb{C}} R / \mathfrak{a}_{r}\left(\mathrm{wt}_{\xi}\right) & =\sum_{m=0}^{r} d_{m}=\sum_{m=0}^{r}\left(a_{0}(\xi) \frac{m^{n-1}}{(n-1) !}+O\left(m^{n-2}\right)\right) \\
& =a_{0}(\xi) \frac{r^{n}}{n !}+O\left(r^{n-1}\right) .
\end{aligned}
$$

By definition of volume in (5), we get the identity.

From now on, we assume that $-\left(K_{E}+\Delta\right) \sim_{\mathbb{Q}} r L$ for $r>0$. We relate the weights on the holomorphic $n$-form to the log-discrepancies. Let $\pi: Y \rightarrow X$ be the extraction (blow-up) of $E$. Then we can write:

$$
K_{Y}+E=\pi^{*}\left(K_{X}\right)+A_{X}(E) E .
$$


$\operatorname{ord}_{E}$ is a valuation on $R$ such that: $\operatorname{ord}_{E}(f)=m$ if $f \in H^{0}\left(E,-m\left(K_{E}+\Delta\right)\right)$.

In the simplest case, $X=C\left(E,-K_{E}\right)$. $X$ has a nonzero vanishing $n$-form given by $\Omega=d z \wedge d w$ where $w$ is the fibre variable $w d z$. The holomorphic vector field $\xi=w \partial_{w}$ has the property that:

$$
\mathcal{L}_{\xi} \Omega=\Omega \text {. }
$$

On the other hand, if we restrict (9) on $E$ we get: $K_{E}=\left.A_{X}(E) E\right|_{E} \Rightarrow A_{X}(E)=1$. More generally, assume that $(E, \Delta)$ is a log-Fano variety such that $-\left(K_{E}+\Delta\right) \sim_{\mathbb{Q}} r \cdot L$. Then the cone $C(E, L)$ is a Calabi-Yau variety with nonvanishing $n$-form $\Omega$. At $w \cdot s$, we have a non-zero holomorphic volume form:

$$
\Omega=d w^{r} \wedge s^{\otimes r}=d w^{r} \wedge \mu_{X}^{*} d z
$$

where $\mu_{X}: Y \rightarrow E$ is the projection. The standard vector $\xi=w \partial_{w}$ satisfies $\mathcal{L}_{\xi} \Omega=r \Omega$. On the other hand, by adjunction, we have: $K_{E}+\Delta_{E}=\left.A_{X}(E) E\right|_{E}=\left.A_{X}(E)(-L)\right|_{E}$. So we get $A_{X}\left(\operatorname{ord}_{E}\right)=A_{X}(E)=r($ see $[$ Kol13, Section 3.1]). Notice that the valuation $\operatorname{ord}_{E}$ coincides with $\mathrm{wt}_{\xi}$.

Remark 2.4. The above discussion can be understood in the setting of the well-known Dolgachev-Pinkham-Demazure construction. In the above we just gave simplified statements to motivate our problem.

So we see that the weight of the action indeed corresponds to log discrepancy. This is also well known in the toric case. Consider a toric affine variety $X_{\sigma}$ defined by a polyhedral cone $\sigma \subset \mathbb{R}^{n}=N \otimes_{\mathbb{Z}} \mathbb{R}$ generated by primitive vectors $v_{1}, \ldots, v_{n} \in N_{\mathbb{Z}}$. Let $\sigma^{\vee} \subset M_{\mathbb{R}}=\left(\mathbb{R}^{n}\right)^{\vee}$ be the dual cone. Assume that there is a rational $\gamma \in \sigma^{\vee} \cap M_{\mathbb{Q}} \subset$ $\left(\mathbb{R}^{n}\right)^{\vee}$ such that

$$
\left\langle\gamma, v_{i}\right\rangle=1 \text {. }
$$

This is equivalent to the condition that $X_{\sigma}$ is $\mathbb{Q}$-Gorenstein. For any rational point $x \in \sigma \cap N_{\mathbb{Q}}, x$ determines a valuation $v_{x}$ such that $v_{x}\left(f_{y}\right)=\langle y, x\rangle$ for any $y \in M_{\mathbb{Z}}$. On the other hand, the toric blow up $\pi_{x}: Y_{x} \rightarrow X$ determined by $x$ is given by the division of $\sigma$ into subcones. There is a unique exceptional divisor $E_{x}$ such that the $\log$ discrepancy is given by (see [Bor97])

$$
A\left(E_{x} ; X\right)=\langle\gamma, x\rangle
$$

which is nothing but the weight of the $\Omega$ with respect to $x$ (see [FOW09, (49)]).

In the study of Sasaki-Einstein metrics, it was Martelli-Sparks-Yau who realized that the problem of minimizing $a_{0}(\xi)$ under the constraint $\mathcal{L}_{\xi} \Omega=n \Omega$ is important because the minimizer is the only candidate of Reeb vector field for which there could exist a Sasaki-Einstein metric (see [MSY08], [GMSY07]). From the above discussion, we see that the problem is the same as minimizing the function $\widehat{\operatorname{vol}}\left(\mathrm{wt}_{\xi}\right)=A_{X}\left(\mathrm{wt}_{\xi}\right)^{n} \operatorname{vol}\left(\mathrm{wt}_{\xi}\right)$ among $\xi \in \mathcal{C} \subset \mathfrak{t}$.

\section{$3 \quad$ Izumi type estimate}

The following inequality will be crucial for us. If $(X, o)$ is smooth, it is well known as shown in [ELS01, Theorem 2.6] and [JM10, Proposition 5.1].

Theorem 3.1 (Izumi type estimate). Assume $(X, o)$ is a $\mathbb{Q}$-Gorenstein klt singularity. There exists a constant $c_{1}=c_{1}(X, o)>0$ such that

$$
v(\mathfrak{m}) \operatorname{ord}_{o} \leq v \leq c_{1} \cdot A_{X}(v) \operatorname{ord}_{o},
$$

for any valuation centered at 0 .

Proof. To get the lower bound, we note that $f \in \mathfrak{m}^{\operatorname{ord}_{o}(f)}-\mathfrak{m}^{\operatorname{ord}_{o}(f)+1}$. So

$$
\operatorname{ord}_{o}(f) v(\mathfrak{m}) \leq v(f) .
$$


For the upper bound, by the way of definition of $A_{X}(v)$ recalled in 2.1 (see [JM10] and [BFFU13]), we can assume that $v$ is divisorial valuation with center at $o$ by approximating the general real valuation by divisorial valuations.

The proof of the upper bound is inspired by [BHJ15, Proof of Theorem 8.13]. When $X$ is smooth, it follows from Skoda's criterion for integrability

$$
\frac{A_{X}(v)}{v(f)} \geq \operatorname{lct}(f)=\min _{v} \frac{A_{X}(v)}{v(f)} \geq \frac{1}{\operatorname{ord}_{o}(f)}
$$

for any $f \in \mathcal{O}_{\mathbf{C}^{n}, 0}$ (see [JM10, Proposition 5.10]). So we have

$$
v(\mathfrak{m}) \operatorname{ord}_{o} \leq v \leq A_{X}(v) \operatorname{ord}_{o}
$$

When $X$ is only klt and $\mathbb{Q}$-Gorenstein, we choose a log resolution $\mu: X^{\prime} \rightarrow X$ and write $\mu^{*} K_{X}=K_{X^{\prime}}+B^{\prime}$ so that:

$$
A_{X}(v)=A_{\left(X^{\prime}, B^{\prime}\right)}(v)=A_{X^{\prime}}(v)-v\left(B^{\prime}\right) .
$$

Because $X$ is klt, we have $B^{\prime} \leq(1-\epsilon) B_{\text {red }}^{\prime}$ for some $\epsilon=\epsilon\left(X^{\prime}\right)>0$. Because $B_{\text {red }}^{\prime}$ has simple normal crossings, we have $\left(X^{\prime}, B_{\text {red }}^{\prime}\right)$ is lc, i.e. $0 \leq A_{\left(X^{\prime}, B_{\text {red }}^{\prime}\right)}(v)=A_{X^{\prime}}(v)-$ $v\left(B_{\text {red }}^{\prime}\right)$. Combining above, we get

$$
\begin{aligned}
A_{X}(v)=A_{X^{\prime}}(v)-v\left(B^{\prime}\right) & =\epsilon A_{X^{\prime}}(v)+(1-\epsilon)\left(A_{X^{\prime}}(v)-v\left(B_{\mathrm{red}}^{\prime}\right)\right)+(1-\epsilon) v\left(B_{\mathrm{red}}^{\prime}\right)-v\left(B^{\prime}\right) \\
& \geq \epsilon A_{X^{\prime}}(v)
\end{aligned}
$$

Assume $\xi$ is the center of $v$ on $X^{\prime}$. Then by the smooth case (see [JM10, Proposition 5.1]), we have:

$$
v(f)=v\left(\mu^{*} f\right) \leq A_{X^{\prime}}(v) \operatorname{ord}_{\xi}\left(\mu^{*} f\right) \leq \epsilon^{-1} A_{X}(v) \operatorname{ord}_{\xi}\left(\mu^{*} f\right) .
$$

By Izumi's linear complementary inequality under morphisms recalled in Theorem 3.2, we have $\operatorname{ord}_{\xi}\left(\mu^{*} f\right) \leq a_{2} \cdot \operatorname{ord}_{o}(f)$ for some $a_{2}=a_{2}(X, o) \geq 1$. So we get the wanted estimate with $c_{1}=\epsilon^{-1} a_{2}$ :

$$
v(\mathfrak{m}) \operatorname{ord}_{o} \leq v \leq \epsilon^{-1} a_{2} \cdot A_{X}(v) \operatorname{ord}_{o} .
$$

In the above proof, we used the following uniform version of Izmui's linear complementary inequalities. In other words, we claimed that $a_{2}$ in the above inequalities can be chosen to be independent of $o^{\prime} \in \mu^{-1}(o)$. Although this should hold in much more generality (see [Izu07, 6]), we just need the following version that follows from Izumi's proof. Notice that if $\xi$ is the center of $v$ on $X^{\prime}$, then $\xi$ is an irreducible subvariety of $X^{\prime}$ and $\operatorname{ord}_{\xi}=\inf _{o^{\prime} \in \xi} \operatorname{ord}_{o}^{\prime}$.

Theorem 3.2 (cf. [Izu82, Izu85]). Let $X$ be a normal affine variety, and $\mu: X^{\prime} \rightarrow X$ be a birational morphism such that $X^{\prime}$ is smooth. Assume that $Z=\mu^{-1}(o)$ is a compact subvariety of $X^{\prime}$. Then there exists a constant $a=a(X, o)$ such that for any $o^{\prime} \in Z$ and any $f \in \mathcal{O}_{X}$ we have

$$
\operatorname{ord}_{o^{\prime}}(\mu \circ f) \leq a \cdot \operatorname{ord}_{o}(f)
$$

Proof. We first recall how Izumi obtained (12) for a fixed $o^{\prime} \in \mu^{-1}(o)$. Izumi showed in [Izu85] that there exist $a_{1}=a_{1}(X, o) \geq 1$ and $b_{1}=b_{1}(X, o) \geq 0$ such that

$$
\left(\mathrm{CI}_{1}\right): \operatorname{ord}_{o}(f g) \leq a_{1}\left(\operatorname{ord}_{o}(f)+\operatorname{ord}_{o}(g)\right)+b_{1}, \text { for any } f, g \in \mathcal{O}_{X, o} .
$$

On the other hand, previously in [Izu82, Theorem 1.2] it was shown that $\left(\mathrm{CI}_{1}\right)$ implies $\left(\mathrm{CI}_{2}\right)$ : there exists $a_{2}=a_{2}\left(o^{\prime}, a_{1}, b_{1}\right) \geq 1$ such that

$$
\left(\mathrm{CI}_{2}\right): \operatorname{ord}_{o^{\prime}}(\mu \circ f) \leq a_{2} \cdot \operatorname{ord}_{o}(f) \text { for any } f \in \mathcal{O}_{X, o} .
$$


To prove that $\left(\mathrm{CI}_{1}\right)$ implies $\left(\mathrm{CI}_{2}\right)$, Izumi first chose a finite surjective morphism $\Pi$ : $(X, o) \rightarrow\left(\mathbb{C}^{n}, 0\right)$ and reduced the proof of $\left(\mathrm{CI}_{2}\right)$ to the following inequality (in [Izu82, Lemma 1.1]), which is the version of the inequality $\left(\mathrm{CI}_{2}\right)$ in the case where the map $(\Pi \circ \mu)$ has both smooth source and target:

$$
\operatorname{ord}_{o^{\prime}}(g \circ \Pi \circ \mu) \leq c \cdot \operatorname{ord}_{0}(g) \text { for any } g \in \mathcal{O}_{\mathbb{C}^{n}, 0} .
$$

In other words, Izumi showed (in [Izu82, Proof of Theorem 1.2]) that $a_{2}=a_{2}\left(a_{1}, b_{1}, c\right)$ for $a_{1}, b_{1}$ in $\mathrm{CI}_{1}$ and $c$ in (13). So we just need to show that $c$, which a priorly depends on $o^{\prime} \in \mu^{-1}(o)$, can be chosen to be uniform with respect to $o^{\prime} \in \mu^{-1}(o) \subset(\Pi \circ \mu)^{-1}(0)$. Since $X^{\prime}$ and $\mathbb{C}^{n}$ are smooth, the inequality (13) was already proved in [Tou80, Lemma 5.6]. We will follow Tougeron's proof to show that the constant $c$ in (13) is indeed uniform.

Choose local coordinates $\left\{z_{j}\right\}_{j=1}^{n}$ around $o^{\prime} \in X^{\prime}$ and let $\left\{w_{i}\right\}_{i=1}^{n}$ be the flat coordinates on $\mathbb{C}^{n}$. Then near $o^{\prime} \in X^{\prime}, \mu$ is locally given by $n$-tuples of holomorphic functions

$$
w_{i}=w_{i}\left(z_{1}, \ldots, z_{n}\right), \quad 1 \leq i \leq n .
$$

Denote the Jacobian matrix by

$$
J=\left(J_{i j}\right)=\left(\frac{\partial w_{i}}{\partial z_{j}}\right)
$$

Because the map $\Pi \circ \mu$ is surjective above a neighborhood of $0 \in \mathbb{C}^{n}$, the Jacobian determinant $\operatorname{det}(J)=\operatorname{det}\left(\partial w_{i} / \partial z_{j}\right)$ is not identically equal to 0 near $o^{\prime} \in X^{\prime}$. Denote $r=r\left(o^{\prime}\right)=\operatorname{ord}_{o^{\prime}} \operatorname{det}(J)$. For any $g \in \mathcal{O}_{\mathbb{C}, 0}$, consider the following system of equations obtained by the chain rule:

$$
\sum_{i=1}^{n}\left(\frac{\partial g}{\partial w_{i}} \circ \mu\right) \frac{\partial w_{i}}{\partial z_{j}}=\frac{\partial}{\partial z_{j}}(g \circ \mu), \quad 1 \leq j \leq n .
$$

If $g \circ \mu \in \mathfrak{m}_{o^{\prime}}^{k+r+1}$, then $\frac{\partial}{\partial z_{j}}(g \circ \mu) \in \mathfrak{m}_{o^{\prime}}^{k+r}$ for any $1 \leq j \leq n$. This implies

$$
\frac{\partial g}{\partial w_{i}} \circ \mu=\operatorname{det}(J)^{-1}(\operatorname{adj}(J))_{i j} \frac{\partial}{\partial z_{j}}(g \circ \mu) \in \mathfrak{m}_{o^{\prime}}^{k}
$$

for any $1 \leq i \leq n$ where $\operatorname{adj}(J)$ is the adjugate matrix of $J$. Iterating this argument, we get that for any multiple index $\alpha \in \mathbb{N}^{n}$ :

$$
\frac{\partial^{\alpha} g}{\partial w^{\alpha}} \circ \mu \in \mathfrak{m}_{o^{\prime}}^{k+r+1-(r+1)|\alpha|} .
$$

On the other hand, if $\operatorname{ord}_{0} g=p$, then there is some multiple index $\beta \in \mathbb{N}^{n}$ with $|\beta|=p$ such that $\frac{\partial^{\beta} g}{\partial w^{\beta}}(0) \neq 0$ and hence $\frac{\partial^{\beta} g}{\partial w^{\beta}} \circ \mu \notin \mathfrak{m}_{o^{\prime}}$. So we get the inequality $k+r+1-(r+1) p \leq 0$, which implies:

$$
\operatorname{ord}_{o^{\prime}}(g \circ \mu) \leq\left(r\left(o^{\prime}\right)+1\right) \cdot \operatorname{ord}_{0} g .
$$

Now we can choose $c=\max \left\{\operatorname{ord}_{o^{\prime}} \operatorname{det}(J) ; o^{\prime} \in(\Pi \circ \mu)^{-1}(0)\right\}$. The maximum can be obtained, because $o^{\prime} \rightarrow \operatorname{ord}_{o^{\prime}} \operatorname{det}(J)$ is upper semicontinuous and $(\Pi \circ \mu)^{-1}(o)$ is a compact set.

Remark 3.3. In Appendix II, we will give a second proof of the needed uniform Izumi estimate in Theorem 3.2 following a referee's suggestion.

Actually there is also a proof of Theorem 3.1 using degeneration argument which involves some deep results from Minimal Model Program (MMP). This method actually allows us to prove a Skoda type estimate, i.e. there exists a constant $c=c(X, o)>0$ such that:

$$
\operatorname{lct}_{o}(f) \geq \frac{c}{\operatorname{ord}_{0}(f)} \text { for any } f \in \mathcal{O}_{X, o} .
$$


Assuming (15), we can easily complete the proof of Theorem 3.1 as in (11). Roughly speaking, the proof of (15) consists of two steps. In the first step one can prove the estimate in the case that $(X, o)$ is an orbifold cone over a log Fano-pair and $f$ is a homogenous function, with the help of uniform estimates of $\alpha$-invariant by Tian and Boucksom-Hisamoto-Jonsson. In the second step one can use the lower semicontinuity of log canonical threshold to reduce the problem to the previous case by considering an equivariant degeneration of any klt singularity to an orbifold cone. It is the degeneration part that is achieved by the MMP through the notion of plt blow-ups. Following a referee's suggestion, to avoid the unnecessary complication involving MMP, we refer the interested reader to the proof presented in an earlier version of this paper on arXiv.

From (10), we immediately get the following inequality:

Corollary 3.4. There exists a constant $c_{1}=c_{1}(X, o)>0$ such that for any $v \in \mathrm{Val}_{X, o}$, we have:

$$
A_{X}(v)^{n} \cdot \operatorname{vol}(v) \geq \frac{e(\mathfrak{m})}{c_{1}^{n}}>0
$$

where $e(\mathfrak{m})$ is the Hilbert-Samuel multiplicity of $R$ along $\mathfrak{m}$.

Remark 3.5. We notice that an inequality closely related to (16) appeared in [dFM15, Theorem 1.3]. However, the Mather version of log discrepancy was used there for general Cohen-Macaulay singularities. In this paper, we use the ordinary log discrepancy for any $\mathbb{Q}$-Gorenstein klt singularities.

\section{Minimizing normalized volume}

From the above discussions in Section 2, it seems natural to ask whether we can minimize the rescaling invariant (0-homogeneous) functional:

$$
\widehat{\operatorname{vol}}(v)= \begin{cases}A_{X}(v)^{n} \operatorname{vol}(v) & \text { if } A_{X}(v)<+\infty \\ +\infty & \text { if } A_{X}(v)=+\infty\end{cases}
$$

To answer this question, we would like some properness property. So we ask whether

$$
\left\{v \in \operatorname{Val}_{X, o} ; A_{X}(v)^{n} \operatorname{vol}(v) \leq M\right\}
$$

is compact under appropriate topology (compare a similar problem in [JM10, Proposition 5.9]). Notice that if $A_{X}(v)=+\infty$, we have defined $\widehat{\operatorname{vol}}(v)=+\infty$.

The main goal in this section is to answer this question positively. The main technical result is the following estimate:

Theorem 4.1. Assume $(X, o)$ is a $\mathbb{Q}$-Gorenstein klt singularity. There exists a positive constant $c_{2}=c_{2}(X, o)>0$ such that for any valuation centered at $o$, we have:

$$
\operatorname{vol}(v) \geq c_{2}\left(\sup _{\mathfrak{m}} \frac{v}{\operatorname{ord}_{o}}\right)^{1-n} \frac{1}{v(\mathfrak{m})} .
$$

In fact, we can choose $c_{2}=2^{-n} e(\mathfrak{m})$ where $e(\mathfrak{m})$ is the Hilbert-Samuel multiplicity of the maximal ideal $\mathfrak{m}$ defining $o \in X$.

Remark 4.2. - Sebastien Boucksom and Mattias Jonsson pointed out to me that there are valuations such that the right hand side is zero. In this case, the inequality (18) becomes trivial. However, if the log discrepancy $A(v)<+\infty$, then Izumi-type inequality in Proposition 3.1 implies that the right hand side of the above inequality is strictly positive.

- $\sup _{\mathfrak{m}}\left(v / \operatorname{ord}_{0}\right)$ is called the "Skewness" function on $v$ in [FJ04]. When $n=2$ and $o=0$ is smooth, by [FJ04, Remark 3.33] (see also [BFJ12, Remark 4.9]), there is an identity

$$
\operatorname{vol}(v)=\left(\sup _{\mathfrak{m}} \frac{v}{\operatorname{ord}_{o}}\right)^{-1} \frac{1}{v(\mathfrak{m})} .
$$


So inequality (18) is a weak generalization of this formula to higher dimension. On the other hand, in higher dimensions, as we will see in Examples 5.1, there is no inequality in the other direction, i.e. there is no uniform upper bound of $\operatorname{vol}(v)\left(\sup _{\mathfrak{m}} v / \operatorname{ord}_{o}\right)^{n-1} v(\mathfrak{m})$.

Corollary 4.3. Theorem 1.1 holds.

Proof. Combining (18) and Izumi type estimate (10), we get the estimate in Theorem 1.1 with $K=c_{2} c_{1}^{1-n}$ :

$$
\begin{aligned}
A_{X}(v)^{n} \cdot \operatorname{vol}(v) & \geq c_{2}\left(\sup _{\mathfrak{m}} \frac{v}{A_{X}(v) \operatorname{ord}_{o}}\right)^{1-n} \frac{A_{X}(v)}{v(\mathfrak{m})} \\
& \geq c_{2} c_{1}^{1-n} \frac{A_{X}(v)}{v(\mathfrak{m})}
\end{aligned}
$$

Now following Jonsson-Mustaţă ([JM10, Section 4.1]), we endow the space $\mathrm{Val}_{X, o}$ with weakest topology for which the evaluation map $\operatorname{Val}_{X, o} \ni v \rightarrow v(f)$ is continuous for all nonzero rational function $f$ in the quotient ring of $R=\mathcal{O}_{X, o}$. To continue, we make the following:

Hypothesis C: $\widehat{\mathrm{vol}}$ is lower semicontinuous on $\mathrm{Val}_{X, o}$.

By Theorem 1.1 we get:

Corollary 4.4. Assume $(X, o)$ is a $\mathbb{Q}$-Gorenstein klt singularity. Under hypothesis $\mathbf{C}$, the normalized volume functional $\widehat{\operatorname{vol}}(v)$ is minimized at a real valuation $v_{*} \in \operatorname{Val}_{X, o}$ (and hence at any positive multiple of $v_{*}$ ).

Proof. By inequality (16), $\widehat{\text { vol }}$ has a positive lower bound on $\mathrm{Val}_{X, o}$. For convenience, we denote:

$$
\widehat{\operatorname{vol}}(X, o):=\inf _{v \in \operatorname{Val}_{X, o}} \widehat{\operatorname{vol}}(v) .
$$

Notice that both sides of (2) are rescaling invariant. So we can restrict to consider only valuations with $v(\mathfrak{m})=1$. By the estimate (2) for any $C>0$, the sub level set

$$
W_{C}:=\left\{v \in \operatorname{Val}_{X} ; c_{X}(v)=o, v(\mathfrak{m})=1, \widehat{\operatorname{vol}}(v) \leq C\right\}
$$

is contained in the following set,

$$
V_{M}:=\left\{v \in \operatorname{Val}_{X} ; c_{X}(v)=o, v(\mathfrak{m})=1, A(v) \leq M\right\}
$$

for some uniform $M=M(C, X, o)>0$. Now using the same proof of [JM10, Proposition $5.9]$, we know that $V_{M}$ is compact subspace of $\operatorname{Val}_{X, o}$. By letting $C \rightarrow \widehat{\operatorname{vol}}(X, o)>0$ (see (19)) and using Hypothesis C, we conclude that there is a minimizer

$$
v_{*} \in \bigcap_{C \geq \widehat{\operatorname{vol}}(X, o)} W_{C} .
$$

Remark 4.5. By [JM10, Lemma 5.7], $A_{X}(v)$ is lower semicontinuous. So if vol is continuous then Hypothesis C is valid. Note that by [BFJ12, Corollary D], vol is continuous on the space of quasi-monomial valuations for a fixed log smooth model. So for any fixed log smooth model $(Y, D)$, vol has a minimizer when restricted to $\mathrm{QM}(Y, D)$. Actually we expect that the minimizer $v_{*}$ is obtained on some log smooth model (item 3 of Conjecture 7.1). 


\subsection{Proof of Theorem 4.1}

Denote by $R=\mathcal{O}_{X, o}$ the local ring at point $o \in X$. The maximal ideal $\mathfrak{m}$ consists of all holomorphic germs vanishing at $o$. Then $R$ is a finitely generated $\mathbb{C}$ algebra and $R / \mathfrak{m}=\mathbb{C}$.

Let $v$ be a valuation centered at $\mathfrak{m}$. From now on we normalize $v$ such that $v(\mathfrak{m})=1$. We will denote $v_{0}=\operatorname{ord}_{o}$. Notice that $v_{0}$ does not have to be a valuation. However it satisfies the following properties:

1. $v_{0}(f g) \geq v_{0}(f)+v_{0}(g)$. The equality does not hold if $\mathrm{Gr}_{m} R$ is not integral (for example: 2-dimensional $A_{2}$-singularity: $R=A_{\mathfrak{m}}$ where $A=\mathbb{C}[x, y, z] /\left(x^{2}+y^{2}+z^{3}\right)$ and $\mathfrak{m}=(\bar{x}, \bar{y}, \bar{z}))$.

2. $v_{0}(f+g) \geq \min \left\{v_{0}(f), v_{0}(g)\right\}$ and the equality holds if $v_{0}(f) \neq v_{0}(g)$.

We define the following invariant of $v$ :

$$
s(v)=\max \left\{2,\left\lceil\sup _{\mathfrak{m}} \frac{v}{v_{0}}\right\rceil\right\} .
$$

It's easy to see that $\left(\operatorname{using} \sup _{\mathfrak{m}}\left(v / v_{0}\right) \geq 1\right)$

$$
s(v) \leq 2 \sup _{\mathfrak{m}}\left(v / v_{0}\right)
$$

By Theorem 3.1, $s=s(v)<\infty$ so that

$$
v_{0}=v(\mathfrak{m}) v_{0} \leq v \leq s(v) v_{0} .
$$

Denote the valuation ideals $\mathfrak{a}_{r}:=\{f \in R ; v(f) \geq r\}$. Then by (22), we have

$$
\mathfrak{m}^{r} \subseteq \mathfrak{a}_{r} \subseteq \mathfrak{m}^{\lfloor r / s\rfloor} .
$$

By (23) we have:

$$
\operatorname{length}\left(R / \mathfrak{m}^{r}\right) \geq \operatorname{length}\left(R / \mathfrak{a}_{r}\right) \geq \operatorname{length}\left(R / \mathfrak{m}^{\lfloor r / s\rfloor}\right) .
$$

Observe that in our situation we can replace length by $\operatorname{dim}_{\mathbb{C}}$, because $\mathfrak{a}_{r}$ is $\mathfrak{m}$-primary. Denote

$$
d_{r}:=\operatorname{length}\left(\mathfrak{m}^{r} / \mathfrak{m}^{r+1}\right)=\operatorname{dim}_{\mathbb{C}}\left(\mathfrak{m}^{r} / \mathfrak{m}^{r+1}\right)=\operatorname{dim}_{\mathbb{C}}\left(R / \mathfrak{m}^{r+1}\right)-\operatorname{dim}_{\mathbb{C}}\left(R / \mathfrak{m}^{r}\right) .
$$

It's well known that:

$$
d_{r}=e(\mathfrak{m}) \frac{r^{n-1}}{(n-1) !}+O\left(r^{n-2}\right)
$$

where $e(\mathfrak{m})$ is the Hilbert-Samuel multiplicity of $\mathfrak{m}$.

Proof of Theorem 4.1. First normalize $v$ such that

$$
1=v(\mathfrak{m})=\inf _{\mathfrak{m}}\{v(f) ; f \in \mathfrak{m}\}=v(g),
$$

for some fixed $g \in \mathfrak{m}$. We will use the notation at the beginning of this section. In particular, $s(v)$ in $(20)$ satisfies $s(v) \leq 2 \sup _{\mathfrak{m}}\left(v / v_{0}\right)$. So we only need to prove the following inequality:

$$
\operatorname{vol}(v) \geq c \cdot s(v)^{1-n},
$$

for some uniform positive constant $c$. For any $l, r \in \mathbb{N}$ with $0 \leq l \leq r$, we denote $p=p(l, s)=\lfloor l / s\rfloor$. Choose a $\mathbb{C}$-basis of $\mathfrak{m}^{p} / \mathfrak{m}^{p+1}$ :

$$
\left[\mathbf{u}^{(p)}\right]:=\left[\left\{u_{1}^{(p)}, \ldots, u_{d_{p}}^{(p)}\right\}\right]=\left\{\left[u_{1}^{(p)}\right], \ldots,\left[u_{d_{p}}^{(p)}\right]\right\} .
$$

We have: $v_{0}\left(u_{i}^{(p)}\right)=p$ for any $1 \leq i \leq d_{p}$. By (22), we have:

$$
p \leq v\left(u_{i}^{(p)}\right) \leq s(v) p .
$$


Now we define the elements:

$$
x_{i}^{(r-l, p)}=g^{r-l} \cdot u_{i}^{(p)}, \text { for } 1 \leq i \leq d_{p} .
$$

Then $v_{0}\left(x_{i}^{(r-l, p)}\right) \geq r-l+p$ and more importantly

$$
v\left(x_{i}^{(r-l, p)}\right)=r-l+v\left(u_{i}^{(p)}\right) \leq r-l+s p=r-l+s\lfloor l / s\rfloor \leq r .
$$

Now we can arrange the following table. Notice that to prove our result, we only need

\begin{tabular}{|c|c|c|c|c|}
\hline$p$ & $l$ & function & lower bound of ord ${ }_{o}$ & range of $v$ \\
\hline 0 & 0 & $x_{1}^{(r, 0)}=g^{r}$ & $r$ & $r$ \\
\hline 0 & 1 & $x_{1}^{(r-1,0)}=g^{r-1}$ & $r-1$ & $r-1$ \\
\hline$\cdots$ & $\cdots$ & $\cdots$ & $\cdots$ & $\cdots$ \\
\hline 0 & $s-2$ & $x_{1}^{(r-s+2,0)}=g^{r-s+2}$ & $r-s+2$ & $r-s+2$ \\
\hline 1 & $s$ & $x_{1}^{(r-s, 1)}, \ldots, x_{d_{1}}^{(r-s, 1)}$ & $r-s+1$ & {$[r-s+1, r]$} \\
\hline 1 & $s+1$ & $x_{1}^{(r-s-1,1)}, \ldots, x_{d_{1}}^{(r-s-1,1)}$ & $r-s$ & {$[r-s, r]$} \\
\hline$\cdots$ & $\cdots$ & 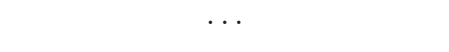 & ( & \\
\hline 1 & $2 s-2$ & $x_{1}^{(r-2 s+2,1)}, \ldots, x_{d_{1}}^{(r-2 s+2,1)}$ & $r-2 s+3$ & {$[r-2 s+3, r]$} \\
\hline 2 & $2 s$ & $x_{1}^{(r-2 s, 2)}, \ldots, x_{d_{2}}^{(r-2 s, 2)}$ & $r-2 s+2$ & {$[r-2 s+2, r]$} \\
\hline$q-1$ & $q s-2$ & $x_{1}^{(r-q s+2, q-1)}, \ldots, x_{d_{q-1}}^{(r-q s+2, q-1)}$ & $r-q s+q+1$ & {$[r-q s+q+1, r]$} \\
\hline$q$ & $q s$ & $x_{1}^{(r-q s, q)}, \ldots, x_{d_{q}}^{(r-q s, q)}$ & $r-q s+q$ & {$[r-q s+q, r]$} \\
\hline$\cdots$ & $\cdots$ & $\cdots$ & $\cdots$ & $\cdots$ \\
\hline$\frac{r}{s}-1$ & $r-2$ & $x_{1}^{(2,(r / s)-1)}, \ldots, x_{d_{(r / s)-2}^{(2,(r / s)-1)}}^{(2, s)}$ & $(r / s)+1$ & {$[(r / s)+1, r]$} \\
\hline$r / s$ & $r$ & $x_{1}^{(0, r / s)}, \ldots, x_{d_{r / s}}^{(0, r / s)}$ & $r / s$ & {$[r / s, r]$} \\
\hline
\end{tabular}
to consider those $r$ 's which are integral multiples of $s$.

We consider the set:

$$
\left\{\left[x_{i}^{(r-l,\lfloor l / s\rfloor)}\right] \in R / \mathfrak{a}_{r+1} \text {; for all } x_{i}^{(r-l,\lfloor l / s\rfloor)} \text { in the 3rd column of the above table }\right\} \text {. }
$$

Claim: The above set consists of linearly independent elements.

Assuming the claim, we can get the estimate of $\operatorname{dim}_{\mathbb{C}} R / \mathfrak{a}_{r+1}$ :

$$
\begin{aligned}
\operatorname{dim}_{\mathbb{C}} R / \mathfrak{a}_{r+1} & \geq(s-1)\left(d_{0}+d_{1}+\cdots+d_{(r / s)-1}\right) \\
& =(s-1) \sum_{i=0}^{(r / s)-1}\left[e(\mathfrak{m}) \frac{i^{n-1}}{(n-1) !}+O\left(i^{n-2}\right)\right] \\
& =\frac{e(\mathfrak{m})(s-1)}{(n-1) !} \frac{1}{n}\left(\frac{r}{s}\right)^{n}+O\left(r^{n-1}\right) \\
& =\frac{e(\mathfrak{m})(s-1)}{s^{n}} \frac{r^{n}}{n !}+O\left(r^{n-1}\right) .
\end{aligned}
$$

So we can estimate the lower bounds of volume:

$$
\operatorname{vol}(v)=\lim _{r \rightarrow+\infty} \frac{n !}{(r+1)^{n}} \operatorname{dim}_{\mathbb{C}} R / \mathfrak{a}_{r+1} \geq e(\mathfrak{m})\left(s^{1-n}-s^{-n}\right) .
$$


By the definition of $s(v)$ in $(20), s \geq 2$ so that $(s-1) / s^{n} \geq s^{1-n} / 2$. Combining this with (21), we get:

$$
\operatorname{vol}(v) \geq e(\mathfrak{m}) s^{1-n} / 2=2^{-n} e(\mathfrak{m})\left(\sup _{\mathfrak{m}} \frac{v}{v_{0}}\right)^{1-n} .
$$

So we complete the proof of Theorem 4.1 with $c_{2}=2^{-n} e(\mathfrak{m})$.

Finally we verify the Claim. Firstly, elements coming from the same row are linearly indepenent. Indeed, for any $\mathbf{a}=\left\{a_{1}, \ldots, a_{d_{p}}\right\} \neq \mathbf{0}$, we have:

$$
x=\sum_{i=1}^{d_{p}} a_{i} x_{i}^{(r-l, p)}=g^{r-l} \sum_{i=1}^{d_{p}} a_{i} u_{i}^{(p)} .
$$

Since $\left\{u_{i}^{(p)} ; 1 \leq i \leq d_{p}\right\}$ is a basis of $\mathfrak{m}^{p} / \mathfrak{m}^{p+1}$, we see that

$$
u^{(p)}:=\sum_{i=1}^{d_{p}} a_{i} u_{i}^{(p)} \in \mathfrak{m}^{p}-\mathfrak{m}^{p+1}
$$

So $\operatorname{ord}_{o}\left(u^{(p)}\right)=p=\lfloor l / s\rfloor$ and $v(x)=r-l+v\left(u^{(p)}\right) \leq r-l+\lfloor l / s\rfloor s \leq r$, which implies $[x] \neq 0 \in R / \mathfrak{a}_{r+1}$.

Next, we show that all elements are linearly independent. Notice that for any linear combination $x$ of all $x_{i}^{(r-l,\lfloor l / s\rfloor)}$ in the 3rd column of the above table, we can decompose it as:

$x=\sum_{l=0, s \nmid(l+1)}^{r} b_{l} x^{(r-l,\lfloor l / s\rfloor)}=\sum_{l=0, s \nmid(l+1)}^{r} b_{l} g^{r-l} u^{(\lfloor l / s\rfloor)}=g^{r-L} \sum_{l=0, s \nmid(l+1)}^{L} b_{l} g^{L-l} u^{(\lfloor l / s\rfloor)}=: g^{r-L} y$,

where $L=\max \left\{l ; b_{l} \neq 0\right\}$ satisfies $s \nmid L+1$, and $u^{(\lfloor l / s\rfloor)}$ is a nontrivial linear combination of $u_{1}^{(\lfloor l / s\rfloor)}, \ldots, u_{d\lfloor l / s\rfloor}^{(\lfloor l / s\rfloor)}$. Now it's clear that (compare with the above table)

$$
v_{0}\left(g^{L-l} u^{(\lfloor l / s\rfloor)}\right) \geq L-l+\lfloor l / s\rfloor>\lfloor L / s\rfloor,
$$

for any $l<L$ and $s \nmid(l+1)$. So we have:

$$
v_{0}(y)=\min \left\{v_{0}\left(g^{L-l} u^{(\lfloor l / s\rfloor)}\right) ; b_{l} \neq 0\right\}=v_{0}\left(u^{(\lfloor L / s\rfloor)}\right)=\lfloor L / s\rfloor .
$$

So we see that

$$
v(x)=r-L+v(y) \leq r-L+s \cdot v_{0}(y)=r-L+s\lfloor L / s\rfloor \leq r,
$$

which implies $[x] \neq 0 \in R / \mathfrak{a}_{r+1}$.

\section{$5 \quad$ Examples}

Example 5.1. We consider monomial valuations on $\left(\mathbb{C}^{n}, 0\right)$. For $\mathbf{x}=\left(x_{1}, \ldots, x_{n}\right) \in$ $\mathbb{R}_{>0}^{n}$, without loss of generality, we can assume $x_{1} \leq x_{2} \leq \cdots \leq x_{n}$.

$$
A_{X}\left(v_{\mathbf{x}}\right)=\sum_{i} x_{i}, \operatorname{vol}\left(v_{\mathbf{x}}\right)=\frac{1}{\prod_{i} x_{i}}, v_{\mathbf{x}}(\mathfrak{m})=\min _{i}\left\{x_{i}\right\}=x_{1}, \sup _{\mathfrak{m}} \frac{v}{\operatorname{ord}_{o}}=\sup _{i}\left\{x_{i}\right\}=x_{n} .
$$

So we get that:

$$
\left(\sup _{\mathfrak{m}} \frac{v}{\operatorname{ord}_{o}}\right)^{n-1} \operatorname{vol}\left(v_{\mathbf{x}}\right) v_{\mathbf{x}}(\mathfrak{m})=\frac{x_{n}^{n-1} x_{1}}{\prod_{i=1}^{n} x_{i}}=\prod_{i=2}^{n-1} \frac{x_{n}}{x_{i}} \geq 1 .
$$

However, there is no upper bound for the right hand side as mentioned in Remark 4.2.

We can also observe the inequalities: 
1 .

$$
\sup _{\mathfrak{m}} \frac{v}{\operatorname{ord}_{o}}=x_{n} \leq \sum_{i=1}^{n} x_{i}=A\left(v_{\mathbf{x}}\right)
$$

2.

$$
A_{X}\left(v_{\mathbf{x}}\right)^{n} \operatorname{vol}\left(v_{\mathbf{x}}\right)=\frac{\left(\sum_{i} x_{i}\right)^{n}}{\prod_{i} x_{i}} \geq \frac{\sum_{i} x_{i}}{\min _{i}\left\{x_{i}\right\}}=\frac{A_{X}\left(v_{\mathbf{x}}\right)}{v_{\mathbf{x}}(\mathfrak{m})}
$$

By using arithmetic-geometric inequality, we also get the estimate:

$$
\operatorname{vol}\left(v_{\mathbf{x}}\right)=\frac{1}{\prod_{i} x_{i}} \geq \frac{n^{n}}{\left(\sum_{i} x_{i}\right)^{n}}
$$

Notice that

$$
\operatorname{lct}\left(\mathfrak{a}_{r}(v)\right)=\frac{1}{r} \sum_{i} x_{i}=\frac{A_{X}\left(v_{\mathbf{x}}\right)}{r}=\frac{A_{X}\left(v_{\mathbf{x}}\right)}{v_{\mathbf{x}}\left(\mathfrak{a}_{r}\left(v_{\mathbf{x}}\right)\right)} \Longrightarrow \operatorname{lct}\left(\mathfrak{a}_{\bullet}\left(v_{\mathbf{x}}\right)\right)=\sum_{i} x_{i} .
$$

So we get:

$$
\operatorname{vol}\left(v_{\mathbf{x}}\right) \geq \frac{n^{n}}{\operatorname{lct}\left(\mathfrak{a}_{\cdot}\left(v_{\mathbf{x}}\right)\right)}
$$

This is essentially the monomial case of more general results from [FEM], [Mus02], which says that:

$$
e\left(\mathfrak{a}_{\bullet}\right) \geq \frac{n^{n}}{\operatorname{lct}\left(\mathfrak{a}_{\bullet}\right)^{n}}
$$

for any graded sequence of zero-dimensional ideals a. in $R$. Applying (25) to $\left\{\mathfrak{a}_{r}(v)\right\}$ we get:

$$
\operatorname{vol}(v) \geq \frac{n^{n}}{\operatorname{lct}(\mathfrak{a} \cdot(v))^{n}} \geq \frac{n^{n}}{A_{X}(v)^{n}}=\frac{A_{X}\left(\operatorname{ord}_{0}\right)^{n} \operatorname{vol}\left(\operatorname{ord}_{0}\right)}{A_{X}(v)^{n}} .
$$

In other words, we have proved the following:

Proposition 5.2. Let $(X, o)=\left(\mathbb{C}^{n}, 0\right)$ and $E=\mathbb{P}^{n-1}$ be the exceptional divisor of the standard blow up at $0 \in \mathbb{C}^{n}$. Then $\operatorname{ord}_{E}$ is a minimizer of $\widehat{\operatorname{vol}}$ on $\mathrm{Val}_{X, o}$ with $\widehat{\operatorname{vol}}\left(\operatorname{ord}_{E}\right)=n^{n}$.

Example 5.3 (experimental example). Assume that $X^{n}=\left\{f\left(z_{1}, \ldots, z_{n+1}\right)=0\right\} \subset$ $\mathbb{C}^{n+1}$ is a hypersurface of dimension $n \geq 3$ with isolated $\mathbb{Q}$-Gorenstein klt singularities at 0 . For any $\mathbf{x}=\left(x_{1}, \ldots, x_{n+1}\right) \in \mathbb{Q}_{+}^{n+1}$, we can rescale it to an $n$-tuple of integers $\tilde{\mathbf{x}} \in \mathbb{Z}_{+}^{n+1}$. Since the normalized volume is rescaling invariant, we will identify $\mathbf{x}$ with $\tilde{\mathbf{x}}$ in this example. hence it determines a weighted blow-up of $\pi=\pi^{\mathbf{x}}: Z=\mathbb{C}_{\mathbf{x}} \rightarrow \mathbb{C}^{n+1}$ with exceptional divisor $F . F$ is the weighted projective space $\mathbb{P}(\mathbf{x})$. Let $Y=X_{\mathbf{x}}$ be the strict transform of $X$ and $\left.\pi\right|_{Y}: Y \rightarrow X$ is $v_{\mathbf{x}}$-blow up with exceptional divisor $E=F \cap Y$. Then $E$ is the hypersurface of weighted degree $v_{\mathbf{x}}(f)$ in $\mathbb{P}(\mathbf{x})$.

$v_{\mathbf{x}}$ induces a valuation on $k\left(X_{\mathbf{x}}\right)$ for which we still denoted by $v_{\mathbf{x}}$ :

$$
v_{\mathbf{x}}(f)=\min _{\left.\tilde{f}\right|_{X_{\mathbf{x}}}=f, \tilde{f} \in k\left(\mathbb{C}_{\mathbf{x}}^{n+1}\right)} v_{\mathbf{x}}(\tilde{f}), \quad f \in \mathbb{C}\left(\mathbb{C}_{\mathbf{x}}^{n+1}\right)
$$

By [Mar96, Lemma 2.2], we have: $v_{\mathbf{x}}(f)=\left\lfloor\frac{1}{d_{\Gamma}} \operatorname{ord}_{\Gamma}(f)\right\rfloor$ where $\Gamma$ is any component of $E=X_{\mathbf{x}} \cap F$ of codimension 2 in $F$ such that $X_{\mathbf{x}}$ is normal at the generic point of $\Gamma$.

Remember that we can assume $\mathbf{x} \in \mathbb{Z}_{+}^{n+1}$ by rescaling. It's easy to see that we have:

$$
K_{Z}+F=\pi^{*} K_{\mathbb{C}^{n+1}}+\left(\sum_{i} x_{i}\right) F, \quad Y=\pi^{*}(X)-v_{\mathbf{x}}(f) F
$$

So we get:

$$
K_{Z}+Y+F=\pi^{*}\left(K_{\mathbb{C}^{n+1}}+X\right)+\left(\left(\sum_{i} x_{i}\right)-v_{\mathbf{x}}(f)\right) F
$$


Taking adjunction, we get:

$$
K_{Y}+\operatorname{Diff}_{Y}(F)=\left(\left.\pi\right|_{Y}\right)^{*} K_{X}+\left.A_{X}\left(v_{\mathbf{x}}\right) F\right|_{Y} .
$$

There are at least two cases one can determine the log discrepancy of the $v_{\mathbf{x}}$ :

- If $Y$ is a Cartier divisor in $Z=\mathbb{C}_{\mathbf{x}}^{n+1}$, $\operatorname{Diff}_{Y}(F)=\left.F\right|_{Y}$ by [Kol13, Proposition 4.2].

- If $\Gamma$ above is not a toric variety, then $A_{X}\left(\frac{1}{d_{\Gamma}} \operatorname{ord}_{\Gamma}\right)=\sum_{i} x_{i}-v_{\mathbf{x}}(f)$ by [Mar96, Proposition 2.3]

The point is that $A_{X}\left(v_{\mathbf{x}}\right)=\sum_{i} x_{i}-v_{\mathbf{x}}(f)$ should hold for generic $\mathbf{x} \in \mathbb{Q}_{+}^{n+1}$. So in this experimental example, we will (continuously) extend this expression to any $\mathbf{x} \in \mathbb{R}_{+}^{n+1}$ and calculate the minimum of $\widehat{\operatorname{vol}}\left(v_{\mathbf{x}}\right)$ as a function of $\mathbf{x} \in \mathbb{R}_{+}^{n+1}$.

1. Consider the $n$-dimensional $A_{1}$ singularity:

$$
X^{n}=\left\{z_{1}^{2}+z_{2}^{2}+\cdots+z_{n}^{2}+z_{n+1}^{2}=0\right\} \subset \mathbb{C}^{n+1} .
$$

By renaming the variables, we consider the weights $\mathbf{x} \in \mathbb{R}_{+}^{n+1}$ satisfying $x_{1} \leq x_{2} \leq$ $\cdots \leq x_{n}$.

$$
A\left(v_{\mathbf{x}}\right)=x_{1}+\cdots+x_{n+1}-2 x_{1},
$$

Modulo $O\left(r^{n-1}\right)$, it's easy to see that:

$$
\operatorname{dim}_{\mathbb{C}} R / \mathfrak{a}_{r}\left(v_{\mathbf{x}}\right)=\frac{1}{\prod_{i=1}^{n+1} x_{i}} \frac{r^{n+1}}{(n+1) !}-\frac{1}{\prod_{i=1}^{n+1} x_{i}} \frac{\left(r-2 x_{1}\right)^{n+1}}{(n+1) !}=\frac{2 x_{1}}{\prod_{i=1}^{n+1} x_{i}} \frac{r^{n}}{n !} .
$$

So $\operatorname{vol}\left(v_{\mathbf{x}}\right)=\frac{2}{\prod_{i=2}^{n+1} x_{i}}$ and we get:

$$
\begin{aligned}
A_{X}\left(v_{\mathbf{x}}\right)^{n} \operatorname{vol}\left(v_{\mathbf{x}}\right) & =\frac{2\left(-x_{1}+\sum_{i=2}^{n+1} x_{i}\right)^{n}}{\prod_{i=2}^{n+1} x_{i}} \geq 2 n^{n}\left(\frac{-x_{1}+\sum_{i=2}^{n+1} x_{i}}{\sum_{i=2}^{n+1} x_{i}}\right)^{n} \\
& \geq 2 n^{n}\left(\frac{-x_{1}+n x_{1}}{n x_{1}}\right)^{n}=2(n-1)^{n} .
\end{aligned}
$$

So $\widehat{\operatorname{vol}}\left(v_{\mathbf{x}}\right)$ obtains the minimum $2(n-1)^{n}$ at $x=(1, \ldots, 1)$.

2. Consider the $n$-dimensional $A_{k-1}$ singularity $(n \geq 2)$ :

$$
X^{n}=A_{k-1}^{n}:=\left\{z_{1}^{2}+z_{2}^{2}+\cdots+z_{n}^{2}+z_{n+1}^{k}=0\right\} \subset \mathbb{C}^{n+1} .
$$

Again for $\mathbf{x}=\left(x_{1}, \ldots, x_{n+1}\right) \in \mathbb{R}^{n+1}$, we can assume $0<x_{1} \leq x_{2} \leq \cdots \leq x_{n+1}$. So we get:

$$
A\left(v_{\mathbf{x}}\right)=\sum_{i=1}^{n+1} x_{i}-\min \left\{2 x_{1}, k x_{n+1}\right\}
$$

There are two cases to consider.

(a) $2 x_{1} \leq k x_{n+1}$. Modulo $O\left(r^{n-1}\right)$ we have:

$$
\operatorname{dim}_{\mathbb{C}} R / \mathfrak{a}_{r}\left(v_{\mathbf{x}}\right)=\frac{1}{\prod_{i=1}^{n+1} x_{i}} \frac{r^{n+1}}{(n+1) !}-\frac{1}{\prod_{i=1}^{n+1} x_{i}} \frac{\left(r-2 x_{1}\right)^{n}}{(n+1) !}=\frac{2 x_{1}}{\prod_{i=1}^{n+1} x_{i}} \frac{r^{n}}{n !} .
$$

So $\operatorname{vol}\left(v_{\mathbf{x}}\right)=2 / \prod_{i=2}^{n+1} x_{i}$ and using arithmetic-geometric mean inequality we can estimate:

$$
\begin{aligned}
\widehat{\operatorname{vol}}\left(v_{\mathbf{x}}\right) & =\left(x_{n+1}+\sum_{i=2}^{n} x_{i}-x_{1}\right)^{n} \frac{2}{x_{n+1} \prod_{i=2}^{n} x_{i}} \\
& \geq 2(n-1)^{n-1}\left(\frac{x_{n+1}+\sum_{i=2}^{n} x_{i}-x_{1}}{\sum_{i=2}^{n} x_{i}}\right)^{n} \frac{\sum_{i=2} x_{i}}{x_{n+1}} .
\end{aligned}
$$


If we define $\alpha=(n-1) x_{n+1} /\left(\sum_{i=2}^{n} x_{i}\right)$ and $\tau=(n-1) x_{1} /\left(\sum_{i=2}^{n} x_{i}\right) \in(0,1)$, then

$$
\widehat{\mathrm{vol}}=2 \frac{(\alpha+(n-1)-\tau)^{n}}{\alpha} \geq 2 \frac{(\alpha+n-2)^{n}}{\alpha}=: \phi(\alpha) .
$$

It's easy to verify that $\phi(\alpha)$ obtains its minimum $\frac{2 n^{n}(n-2)^{n-1}}{(n-1)^{n-1}}$ at $\alpha_{*}=\frac{n-2}{n-1}$, is strictly decreasing on $\left(0, \alpha_{*}\right)$ and strictly increasing on $\left(\alpha_{*},+\infty\right)$. Tracking the equality case of the above estimates, we get that $\widehat{\mathrm{vol}}$ obtains $\phi\left(\alpha_{*}\right)$ only if $\mathbf{x}_{*}=\left(1,1, \ldots, 1, \frac{n-2}{n-1}\right)$. The constraint is satisfied at the minimizer $\mathbf{x}_{*}$ if and only if $2 \leq k \frac{(n-2)}{n-1}$.

(b) $2 x_{1} \geq k x_{n+1}$. In this case,

$$
A\left(v_{\mathbf{x}}\right)=\sum_{i=1}^{n} x_{i}+x_{n+1}-k x_{n+1}, \quad \operatorname{vol}\left(v_{\mathbf{x}}\right)=\frac{k}{\prod_{i=1}^{n} x_{i}} .
$$

So

$$
\widehat{\operatorname{vol}}\left(v_{\mathbf{x}}\right)=\frac{k\left(\sum_{i=1}^{n} x_{i}-(k-1) x_{n+1}\right)^{n}}{\prod_{i=1}^{n} x_{i}} \geq k n^{n}\left(\frac{\sum_{i=1}^{n} x_{i}-(k-1) x_{n+1}}{\sum_{i=1}^{n} x_{i}}\right)^{n} .
$$

The last expression is a decreasing function of $x_{n+1}$ and hence obtains the minimum at $x_{n+1}=2 x_{1} / k$ which is the right end point of the interval $\left(0,2 x_{1} / k\right)$ for $x_{n+1}$ in this case. So $\widehat{\text { vol }}$ obtains its minimum $\frac{((n-2) k+2)^{n}}{k^{n-1}}=\phi(2 / k)$ at the weight $\left(1,1, \ldots, 1, \frac{2}{k}\right)$ under the constraint $2 x_{1} \geq k x_{n+1}$.

Combining the above discussions, we see that if $2 \leq k \frac{n-2}{n-1}$, then $\widehat{\operatorname{vol}}\left(v_{\mathbf{x}}\right)$ obtains the minimum $\phi\left(\alpha_{*}\right)$ at weight $\left(1, \ldots, 1, \frac{n-2}{n-1}\right)$. Otherwise, the minimum is $\phi(2 / k)$ and is obtained at the weight $(1, \ldots, 1,2 / k)$. We can organize the situation in the following table.

\begin{tabular}{|c|c|c|c|c}
\hline \multicolumn{1}{c|}{$n$} & 2 & 3 & 4 & $n \geq 5$ \\
\hline 1 & $(1,1,2)$ & $(1,1,1,2)$ & $(1,1,1,1,2)$ & $(1, \ldots, 1,2)$ \\
2 & $(1,1,1)$ & $(1,1,1,1)$ & $(1,1,1,1,1)$ & $(1, \ldots, 1,1)$ \\
\cline { 1 - 1 } 3 & $(1,1,2 / 3)$ & $(1,1,1,2 / 3))$ & $(1,1,1,1,2 / 3)$ & $\left(1, \ldots, 1, \frac{n-2}{n-1}\right)$ \\
4 & $(1,1,1 / 2)$ & $(1,1,1,1 / 2)$ & $(1,1,1,1,2 / 3)$ & $\left(1, \ldots, 1, \frac{n-2}{n-1}\right)$ \\
\cline { 3 - 3 } & & $(1,1,1,1 / 2)$ & $(1,1,1,1,2 / 3)$ & $\left(1, \ldots, 1, \frac{n-2}{n-1}\right)$ \\
\hline
\end{tabular}

Surprisingly, this table matches a table (pointed to us by H-J. Hein) coming out of the study of Ricci-flat Kähler cone metrics or the closely related Sasaki-Einstein metrics. This motivates one of our conjectures in Conjecture 7.1. For comparison and for the reader's convenience, we give some discussion analogous to that in [DS15, Section 3.3]. We denote by $A_{k-1}^{n}$ the $n$-dimensional $A_{k-1}$ singularities considered above. Then we have the following cases:

(a) $n=2$. For any $k \geq 1, A_{k-1}^{2} \cong \mathbb{C}^{2} / \mathbb{Z}_{k-1}$ has a flat quotient metric.

(b) $k=1$. For any $n \geq 2, A_{0}^{n}=\mathbb{C}^{n}$ has the standard flat metric.

(c) $k=2$. For any $n \geq 2, A_{1}^{n}$ has a rotationally symmetric Ricci-flat cone metric which could be obtained by solving a simple ODE using Calabi's ansatz.

(d) $(n, k)=(3,3)$. There is a Ricci-flat cone Kähler metric with the weight of the $\mathbb{C}^{*}$-action given by $(1,1,1,2 / 3)$. This is nontrivial and was first proved in [LS14] (see also [Li15a]). 
(e) $(n, k)=(3,4)$ or $(4,3)$. For these two special weights (boxed weights in the table), the corresponding singularities are K-semistable but not K-polystable. More precisely, if $D^{2}$ (resp. $D^{3}$ ) denotes the smooth quadric hypersurface in $\mathbb{P}^{2}$ (resp. $\left.\mathbb{P}^{3}\right)$, then the associated pair $\left(\mathbb{P}^{n-1},\left(1-\frac{1}{k}\right) D\right)$ is log-K-semistable but not log-K-polystable by [LS14, Li13].

(f) For all the other cases (corresponding to all the weights below the short horizontal segments), we have $2<k \frac{n-2}{n-1}$. It was shown in [GMSY07] that there is no (obvious) Sasaki-Einstein metric on the corresponding $A_{k-1}^{n}$ singularity. On the other hand, Hein-Naber recently showed in [HN] that there is a local (non-conic) Ricci-flat metric near the vertex $o \in A_{k-1}^{n}$ whose metric tangent cone at $o$ is $A_{1}^{n-1} \times \mathbb{C}$ with the product Ricci flat metric. The

Kähler potential of the Ricci flat metric is given by $\left(\left|z_{1}\right|^{2}+\cdots+\left|z_{n}\right|^{2}\right)^{\frac{n-2}{n-1}}+$ $\left|z_{n+1}\right|^{2}$. It was then observed in [DS15] that the weights $\left(\frac{n-1}{n-2}, \ldots, \frac{n-1}{n-2}, 1\right)=$ $\frac{n-1}{n-2}\left(1, \ldots, 1, \frac{n-2}{n-1}\right)$ indeed degenerates $A_{k-1}^{n}$ into $A_{1}^{n-1} \times \mathbb{C}$ in these cases, i.e. when $k>2 \frac{n-1}{n-2}$.

In the Appendix I, we calculate the candidate minimizing valuations for D-type and E-type singularities

\section{Relation to Fujita's divisorial stability}

In this section, we carry out calculations to show that there is a close relation between minimization of $\widehat{\text { vol }}$ with Fujita's divisorial stability ([Fuj15a]) which is a consequence of K-stability ([Tia97], [Don02], see also [Ber12]). The calculations will essentially show that the derivative of $\widehat{\text { vol }}$ at the canonial valuation on the cone along some directions of $\mathbb{C}^{*}$-invariant valuations is given by Fujita's invariant on the base. Note that Fujita's invariant is an example of CM weight ([Tia97], [Don02]) which is a generalization of the orginal Futaki invariant ([Fut83]) to the setting of degenerations. So our calculation is a reflection of the calculation of Martelli-Sparks-Yau (see [MSY08]) by which they showed that the derivative of the (normalized) volume function at a regular Reeb vector field is the classical Futaki invariant. Although this point will be developed in more generality in [Li15b], to the author the calculation here was an initial evidence of the validity of our theory beyond the motivations from Sasaki geometry recalled earlier.

From now on let $V$ be a $\mathbb{Q}$-Fano variety with $\mathbb{Q}$-Gorenstein klt singularities. We first recall Fujita's divisorial semistability.

Definition 6.1 ([Fuj15a, Definition 1.1]). Let D be a nonzero effective Weil divisor on $V$. The pair $\left(V,-K_{V}\right)$ is said to be divisorial semistable along $D$ if the value:

$$
\eta(D)=\operatorname{Vol}_{V}\left(-K_{V}\right)-\int_{0}^{+\infty} \operatorname{Vol}_{V}\left(-K_{V}-x D\right) d x
$$

satisfies $\eta(D) \geq 0$, where $\mathrm{Vol}_{V}$ is the volume function on divisors (see [Laz96]).

Assume $-K_{V} \sim_{\mathbb{Q}} r L$ for some Cartier divisor $L$ and $r>0$. Let $X=C(V, L)$ be the affine cone over $V$ with polarization $L$. Let $\pi_{0}: W=B l_{o} X \rightarrow X$ be the blow-up of $X$ at $o$ with the exceptional divisor still denoted by $V$. Let $D$ be a prime divisor on $V$. We think $D$ as an irreducible divisor contained in the exceptional divisor $V$ and consider the blow up $\pi_{1}: Y:=B l_{D} W \rightarrow W$ with the exceptional divisor $E_{1}$. Let $\widehat{V}$ or $E_{0}$ denote the strict transform of $V$. Then $\pi:=\pi_{1} \circ \pi_{0}:\left(Y, \widehat{V}+E_{1}\right) \rightarrow(X, o)$ is a birational morphism and there are two divisorial valuations assocated to $\widehat{V}$ and $E_{1}$ respectively. We will compare the normalized volume of these two valuations. For $v_{0}:=\operatorname{ord}_{\widehat{V}}=\operatorname{ord}_{V}$, it's easy to see that

$$
\operatorname{vol}\left(v_{0}\right)=L^{n-1}
$$

For $v_{1}:=\operatorname{ord}_{E_{1}}$, by similar calculations as in [Kur03, (11)] and [Fuj15a] (see also [Li15b]), we get (compare (29)) 
Lemma 6.2.

$$
\operatorname{vol}\left(v_{1}\right)=L^{n-1}-n \int_{0}^{+\infty} \operatorname{Vol}(L-x D) \frac{d x}{(1+x)^{n+1}} .
$$

To find the log discrepancy, we notice that:

$$
K_{W}=\pi_{0}^{*} K_{X}+(r-1) V, \quad K_{Y}=\pi_{1}^{*} K_{W}+E_{1}, \quad \pi_{1}^{*} V=\widehat{V}+E_{1} .
$$

Recall that $E_{0}=\widehat{V}$ is the strict transform of $V$ under $\pi_{1}$. The second identity is because $D$ is codimension 2 inside $W$. So we get:

$$
K_{W}=\pi^{*} K_{X}+(r-1) \widehat{V}+r E_{1} .
$$

So we get $A_{X}(V)=r$ and $A_{X}\left(E_{1}\right)=r+1$.

Proposition 6.3. In the above setting, the following statements hold.

1. If $\operatorname{ord}_{V}$ minimizes $\widehat{\mathrm{vol}}$, then $V$ is divisorial semistable along any prime divisor $D$.

2. If $V$ is divisorial semistable along a prime divisor $D$, then $\widehat{\operatorname{vol}}\left(\operatorname{ord}_{E_{1}}\right) \geq \widehat{\operatorname{vol}}\left(\operatorname{ord}_{V}\right)=$ $L^{n-1}$, where $E_{1}$ is the exceptional divisor over $o \in X=C(V, L)$ associated to $D$.

Proof. To compare the two normalized volume, we will first calculate the normalized volume function for quasi-monomial valuations on $\left(Y, \widehat{V}+E_{1}\right)$. By section 2.1, for any vector $(\alpha, 1)$ with $\alpha>0$, we have a valuation $w_{\alpha}$ satisfying:

1. $w_{\alpha}$ can be defined by the following condition:

$$
w_{\alpha}(f) \geq \alpha \cdot i+(i+j) \text {, for any } f \in H^{0}(V, i L-j D) .
$$

The identity holds if and only if $f \notin H^{0}(V, i L-(j+1) D)$.

2. $w_{\alpha}$ interpolates $v_{0}$ and $v_{1}$ in the normalized sense:

$$
\lim _{\alpha \rightarrow+\infty} \frac{w_{\alpha}}{\alpha}=v_{0}, \quad \lim _{\alpha \rightarrow 0} w_{\alpha}=v_{1}
$$

From item 1 above, we know that $\mathfrak{a}_{\alpha \cdot i+(i+j)}\left(w_{\alpha}\right) \cap H^{0}(i L)=H^{0}(i L-j D)$. Notice that $w_{\alpha}$ are all $\mathbb{C}^{*}$-invariant valuations. So the co-length of $\mathfrak{a}_{m+1}\left(w_{\alpha}\right)$ is equal to:

$$
\sum_{i=0}^{m /(\alpha+1)}\left(h^{0}(i L)-h^{0}(i L-(m-(\alpha+1) i) D)\right) .
$$

It's easy to see that, modulo $m^{n-1}$, we have:

$$
n ! \sum_{i=0}^{m /(\alpha+1)} h^{0}(i L)=L^{n-1}\left(\frac{m}{\alpha+1}\right)^{n} .
$$

For the other sum, we calculate it following the method in [Fuj15a]. Let $\tau(D)$ be the pseudo-effective threshold of $D$ with respect to $(V, L)$. Then because $V$ (or its projective small $\mathbb{Q}$-factorial modification) is a Mori dream space (see [Fuj15a, Lemma 2.3]), by [KKL12, Theorem 4.2] (see [Fuj15a, Theorem 2.5]), there exist

1. an increasing sequence of rational numbers

$$
0=\tau_{0}<\tau_{1}<\cdots<\tau_{N}=\tau(D),
$$

2. a normal projective varieties $V_{1}, \ldots, V_{N}$, and

3. mutually distinct birational contraction maps $\phi_{k}: V \rightarrow V_{k}$ such that the following hold:

1. for any $x \in\left[\tau_{k-1}, \tau_{k}\right)$, the map $\phi_{k}$ is a semiample model of $L-x D$, and

2. if $x \in\left(\tau_{k-1}, \tau_{k}\right)$, then the map $\phi_{k}$ is the ample model of $L-x D$. 
For $0 \leq k \leq N$, we define the thresholds $I(k)=m /\left(\alpha+1+\tau_{k}\right)$ such that

$$
\frac{m}{\alpha+1}=I(0)>I(1)>\cdots \geq I(N)>I(N+1)=: 0 .
$$

If $I(k+1) \leq i \leq I(k)$ (resp. $I(k+1)<i<I(k))$, then $(m-(\alpha+1) i) / i \in\left[\tau_{k}, \tau_{k+1}\right]$ (resp. $\left.\left(\tau_{k}, \tau_{k+1}\right)\right)$ so that $i L-(m-(\alpha+1) i) D=i\left(L-\frac{m-(\alpha+1) i}{i} D\right)$ is semiample (resp. ample) on $V_{k}$. Then modulo $m^{n-1}$, we have (see [Fuj15a, Section 4, 5]):

$$
\begin{aligned}
& n ! \sum_{i=1}^{m /(\alpha+1)} h^{0}(V, i L-(m-(\alpha+1) i) D) \\
= & n ! \sum_{k=0}^{N-1} \sum_{i=\lfloor I(k+1)\rfloor}^{\lfloor I(k)\rfloor-1} h^{0}\left(V_{k}, i L_{k}-(m-(\alpha+1) i) D_{k}\right)+O\left(m^{n-1}\right) \\
= & n ! \sum_{k=0}^{N-1} \sum_{i=\lfloor I(k+1)\rfloor}^{\lfloor I(k)\rfloor-1} \chi\left(V_{k}, i L_{k}-(m-(\alpha+1) i) D_{k}\right)+O\left(m^{n-1}\right) \\
= & n \sum_{k=0}^{N-1} \int_{I(k+1)}^{I(k)}\left(s L_{k}-(m-(\alpha+1) s) D_{k}\right)^{n-1} d s+O\left(m^{n-1}\right) \\
= & n \sum_{k=0}^{N-1} \int_{I(k)}^{I(k+1)} \operatorname{Vol}_{V}(s L-(m-(\alpha+1) s) D)^{n-1} d s+O\left(m^{n-1}\right) \\
= & n \int_{0}^{\frac{m}{\alpha+1}} \operatorname{Vol}_{V}(s L-(m-(\alpha+1) s) D) d s+O\left(m^{n-1}\right) \\
= & m^{n} n \int_{0}^{+\infty} \operatorname{Vol}_{V}(L-x D) \frac{d x}{(\alpha+1+x)^{n+1}} .
\end{aligned}
$$

So we get:

$$
\operatorname{vol}\left(w_{\alpha}\right)=\frac{L^{n-1}}{(\alpha+1)^{n}}-n \int_{0}^{+\infty} \operatorname{Vol}(L-x D) \frac{d x}{(\alpha+1+x)^{n+1}} .
$$

Notice that $(1+\alpha)^{n} \operatorname{vol}\left(w_{\alpha}\right)=\operatorname{vol}\left(w_{\alpha} /(1+\alpha)\right)$ interpolates between (26) and (27).

For the log discrepancy of $w_{\alpha}$, by the definition (8) in Section 2.1 and (28), we get:

$$
A\left(w_{\alpha}\right)=\alpha r+(r+1) .
$$

So we get the normalized volume function:

$$
\begin{aligned}
\widehat{\operatorname{vol}}\left(w_{\alpha}\right) & =A\left(w_{\alpha}\right)^{n} \cdot \operatorname{vol}\left(w_{\alpha}\right) \\
& =\frac{(\alpha r+(r+1))^{n}}{(\alpha+1)^{n}} L^{n-1}-n \int_{0}^{\infty} \operatorname{Vol}(L-x D) \frac{(\alpha r+(r+1))^{n}}{(\alpha+1+x)^{n+1}} d x \\
& =\left(\frac{r+(r+1) \beta}{1+\beta}\right)^{n} L^{n-1}-n \beta \int_{0}^{\infty} \operatorname{Vol}(L-x D) \frac{(r+(r+1) \beta)^{n}}{(1+(1+x) \beta)^{n+1}} d x \\
& =: \Phi(\beta),
\end{aligned}
$$

where we substituted $\beta=\alpha^{-1}$ such that $\Phi(0)=\widehat{\operatorname{vol}}\left(v_{0}\right)$ and $\Phi(+\infty)=\widehat{\operatorname{vol}}\left(v_{1}\right)$. We calculate the directional derivative $\Phi^{\prime}(0)$ :

$$
\begin{aligned}
\Phi^{\prime}(0) & =n r^{n-1} L^{n-1}-n r^{n} \int_{0}^{\infty} \operatorname{Vol}(L-x D) d x \\
& =n\left(\left(-K_{V}\right)^{n-1}-\int_{0}^{\infty} \operatorname{Vol}\left(-K_{V}-x D\right) d x\right) .
\end{aligned}
$$

Notice that the expression in the last bracket is exactly Fujita's invariant $\eta(D)$ in Definition 6.1. So if $\eta(D)<0$, then $\Phi^{\prime}(0)<0$ so that $\widehat{\operatorname{vol}}\left(w_{\alpha}\right)<\widehat{\operatorname{vol}}\left(v_{0}\right)$ if $\beta=\alpha^{-1}$ is 
sufficiently small. This contradicts the assumption that $\widehat{\operatorname{vol}}\left(v_{0}\right)$ is the minimum. So we get the first statement of Theorem 6.3.

To see the second statement, we can write

$$
\frac{(\alpha, 1)}{\alpha r+r+1}=\frac{\alpha r}{\alpha r+r+1} \frac{(1,0)}{r}+\frac{r+1}{\alpha r+r+1} \frac{(0,1)}{r+1}=:(1-t) \frac{(1,0)}{r}+t \frac{(0,1)}{r+1},
$$

where we introduced a new variable $t=\frac{r+1}{\alpha r+r+1}$, or equivalently $\alpha=\beta^{-1}=\frac{1-t}{t} \frac{r+1}{r}$. So we get $w_{\alpha} /(\alpha r+r+1)=: \tilde{v}_{t}$ is a "linear" interpolation between $\tilde{v}_{0}=v_{0} / r$ and $\tilde{v}_{1}=v_{1} /(r+1)$. Notice that $A\left(\tilde{v}_{t}\right) \equiv 1$.

Denote $f(t):=\widehat{\operatorname{vol}}\left(\tilde{v}_{t}\right)=\Phi(\beta(t))$. The directional derivative of $f(t)$ at $t=0$ is calculated by the chain rule:

$$
f^{\prime}(0)=\Phi^{\prime}(0) \beta^{\prime}(0)=n \cdot \eta(D) \cdot \frac{r}{r+1} .
$$

We claim that $f(t)$ is a convex function of $t$. Assuming the claim, we know that $f^{\prime}(0) \geq 0$ implies $f(1) \geq f(0)$. So we obtain the second statement of 6.3 . To verify the claim, we re-write the formula of $\Phi(\beta)$ in (30) by the integration by parts:

$$
\begin{aligned}
\frac{\Phi(\beta)}{(r+(r+1) \beta)^{n}} & =\frac{L^{n}}{(1+\beta)^{n}}+\left.\frac{\operatorname{Vol}(L-x D)}{(1+(1+x) \beta)^{n}}\right|_{x=0} ^{+\infty}-\int_{0}^{+\infty} \frac{d \operatorname{Vol}(L-x D)}{(1+(1+x) \beta)^{n}} \\
& =-\int_{0}^{+\infty} \frac{d \operatorname{Vol}(L-x D)}{(1+(1+x) \beta)^{n}} .
\end{aligned}
$$

So, with $\beta=\frac{t r}{(1-t)(r+1)}$, we easily get:

$$
f(t)=\Phi(\beta(t))=r^{n}(r+1)^{n} \int_{0}^{+\infty} \frac{-d \operatorname{Vol}(L-x D)}{(r+1+(r x-1) t)^{n}} .
$$

Because $\operatorname{Vol}(L-x D)$ is a decreasing function of $x \in[0,+\infty),-d \operatorname{Vol}(L-x D)$ is a measure with positive density with respect to the Lebesgue measure $d x$. So the claim follows from the fact that $t \mapsto(r+1+(r x-1) t)^{-n}$ is a convex function on $[0,1]$.

\section{Questions and discussions}

\subsection{Some conjectures}

There are several natural questions that deserve further studies. We collect them into following conjectures which we plan to study. The calculations in this paper should be viewed as evidences for these conjectures.

Conjecture 7.1. 1. Hypothesis $\mathbf{C}$ is true. As a consequence, for any germ of $\mathbb{Q}$-Gorenstein klt singularity $(X, o)$, there exists a minimizer (denoted by $v_{*}=$ $\left.v_{*}(X, o)\right)$ of $\widehat{\operatorname{vol}}(v)$ by Corollary 4.4 .

2. $v_{*}$ is unique up to positive rescaling.

3. $v_{*}$ is always a quasi-monomial valuation.

4. Let $V$ be a Fano manifold, and let $X=C\left(V,-K_{V}\right)$. Then $V$ is K-semistable if and only if on the cone singularity $(X, o), \widehat{\operatorname{vol}}(v)$ is minimized at $\operatorname{ord}_{V}$ where $V$ is considered as the exceptional divisor of the blow up $\pi: B l_{o} X \rightarrow X$.

5. Assume $(X, o)$ is a $\mathbb{Q}$-Gorenstein klt singularity on a Kähler-Einstein Fano variety $\left(X, \omega_{\mathrm{KE}}\right)$. The minimizer $v_{*}(X, o)$ for $(X, o)$ is exactly the weight function that gives the metric tangent cone at $o \in\left(X, d_{\omega_{\mathrm{KE}}}\right)$. More precisesly, we consider the the associated graded algebra of $v_{*}$ :

$$
\operatorname{gr}_{v_{*}} R=\bigoplus_{m \in \Phi} \mathfrak{a}_{m}\left(v_{*}\right) / \mathfrak{a}_{>m}\left(v_{*}\right),
$$


where $\Phi$ is the valuation semigroup of $v_{*}$. Then the conjecture is that $\operatorname{gr}_{v_{*}} R$ is finitely generated and normal, and $\operatorname{Spec}\left(\operatorname{gr}_{v_{*}} R\right)$ specially degenerates to the metric tangent cone at $(X, o)$. If true, this is an answer to a question of Donaldson-Sun [DS15].

6. For (Newton non-degenerate) hypersurface klt singularities in $\mathbb{C}^{n+1}$, the global minimizers of $\widehat{\mathrm{vol}}$ can be found among the valuations induced by weighted blow ups of the ambient $\mathbb{C}^{n+1}$.

Postscript Note: After the initial writing of this paper, there have been progresses on the above conjectures (see [Li15b, LL16, Blu16]). In particular Blum proved the existence of minimizers without verifying the lower semicontinuity of $\widehat{\mathrm{vol}}$ (but using the main estimates in this paper). However the quasi-monomial part and uniqueness part are still open in general.

\section{Appendix I: Candidate minimizer for D-type and E-type singularities}

1. Consider the $(n+1)$-dimensional $D_{k+1}$ singularity:

$$
X^{n+1}=D_{k+1}^{n+1}:=\left\{z_{1}^{2}+\cdots+z_{n}^{2}+z_{n+1}^{2} z_{n+2}+z_{n+2}^{k}=0\right\} \subset \mathbb{C}^{n+2} .
$$

with $n \geq 1$ and $k \geq 3$.

Consider the valuation determined by $\mathbf{x}=\left(x_{1}, \ldots, x_{n}, x_{n+1}, x_{n+2}\right)$ with $x_{1} \ldots x_{n}$. Then

$A_{X}\left(v_{\mathbf{x}}\right)=\sum_{i=1}^{n+2} x_{i}-\min \left\{2 x_{1}, 2 x_{n+1}+x_{n+2}, k x_{n+2}\right\}, \operatorname{vol}\left(v_{\mathbf{x}}\right)=\frac{\min \left\{2 x_{1}, 2 x_{n+1}+x_{n+2}, k x_{n+2}\right\}}{\prod_{i=1}^{n+2} x_{i}}$.

The minimization of $\widehat{\operatorname{vol}}\left(v_{\mathbf{x}}\right)$ is a standard multivariable calculus problem. Although the complete discussion is messy, the end results are clean and are orgainized in the following table.

\begin{tabular}{|c|c|c|c|c}
\hline \multicolumn{1}{|c|}{2} & 2 & 3 & 4 & $\geq 5$ \\
\hline 3 & $\left(1, \frac{2}{3}, \frac{2}{3}\right)$ & $\left(1,1, \frac{2}{3}, \frac{2}{3}\right)$ & $\left(1,1,1, \frac{2}{3}, \frac{2}{3}\right)$ & $\left(1, \ldots, 1, \frac{n-2}{n-1}, \frac{n-2}{n-1}\right)$ \\
$k \geq 4$ & $\left(1, \frac{k-1}{k}, \frac{2}{k}\right)$ & $\left(1,1, \alpha_{*}(2), 2-2 \alpha_{*}(2)\right)$ & $\left(1,1,1, \alpha_{*}(3), 2-2 \alpha_{*}(3)\right)$ & $\left(1, \ldots, 1, \frac{n-2}{n-1}, \frac{n-2}{n-1}\right)$ \\
\hline
\end{tabular}

Here $\alpha_{*}(n)=\frac{-n+\sqrt{5 n^{2}-4 n}}{2(n-1)}: \alpha_{*}(2) \approx 0.732, \alpha_{*}(3) \approx 0.686$. Denoting the weights in the above table by $w_{*}$, we have the following cases of associated degenerations:

(a) $k=3$ and $2 \leq n+1 \leq 5$, or $n+1=2$ and any $k$, the weight in the above table comes from a natural $\mathbb{C}^{*}$-action preserving $X$. The corresponding normalized volume $\widehat{\operatorname{vol}}\left(w_{*}\right)=\frac{((n-1) k+1)^{n+1}}{k^{n-1}(k-1)}$.

(b) For $n+1=3,4$ and $k \geq 4$ (irrational $w_{*}$ with $\widehat{\operatorname{vol}}\left(w_{*}\right)=\phi_{2}\left(\alpha_{*}\right)$ ), or $n+1=5$ and $k \geq 4\left(w_{*}=\left(1, \ldots, 1, \frac{2}{3}, \frac{2}{3}\right)\right.$ with $\left.\widehat{\operatorname{vol}}\left(w_{*}\right)=\frac{(3 n-2)^{n+1}}{2 \cdot 3^{n-1}}\right)$, the corresponding weight "degenerates" $X$ to the (non-isolated) singularity given by

$$
Y^{n+1}=\left\{z_{1}^{2}+\cdots+z_{n}^{2}+z_{n+1}^{2} z_{n+2}=0\right\} \subset \mathbb{C}^{n+2} .
$$

(c) For any other case $(n+1 \geq 6$ and $k \geq 3)$, the weight $w_{*}=\left(1, \ldots, 1, \frac{n-2}{n-1}, \frac{n-2}{n-1}\right)$, with $\widehat{\operatorname{vol}}\left(w_{*}\right)=2(n+1)^{n+1} \frac{(n-2)^{n-1}}{(n-1)^{n-1}}$, degenerates $X$ to the singularity:

$$
A_{1}^{n-1} \times \mathbb{C}^{2}=\left\{z_{1}^{2}+\cdots+z_{n}^{2}=0\right\} \subset \mathbb{C}^{n+2} .
$$


2. Consider the $(n+1)$-dimensional $E_{7}$ singularity:

$$
X^{n+1}=E_{7}^{n+1}:=\left\{z_{1}^{2}+z_{2}^{2}+\cdots+z_{n}^{2}+z_{n+1}^{3} z_{n+2}+z_{n+2}^{3}=0\right\} \subset \mathbb{C}^{n+2} .
$$

By renaming the variables, we can rearrange the weight $\mathbf{x}$ such that $x_{1} \leq x_{2} \leq$ $\cdots \leq x_{n}$. So the problem is to minimize the functional:

$$
\widehat{\operatorname{vol}}\left(v_{\mathbf{x}}\right)=\left(\sum_{i=1}^{n+2} x_{i}-\min \left\{2 x_{1}, 3 x_{n+1}+x_{n+2}, 3 x_{n+2}\right\}\right)^{n+1} \frac{\min \left\{2 x_{1}, 3 x_{n+1}+x_{n+2}, 3 x_{n+2}\right\}}{\prod_{i=1}^{n+2} x_{i}}
$$

We get:

(a) $n \geq 5$. The unique minimizer is the weight $w_{*}=\left(1, \ldots, 1, \frac{n-2}{n-1}, \frac{n-2}{n-1}\right)$ with $\widehat{\operatorname{vol}}\left(w_{*}\right)=2(n+1)^{n+1} \frac{(n-2)^{n-1}}{(n-1)^{n-1}}$, degenerating $X$ to $A_{1}^{n-1} \times \mathbb{C}^{2}=\left\{z_{1}^{2}+\cdots+z_{n}^{2}=\right.$ $0\} \subset \mathbb{C}^{n+2}$. Notice that $A_{1}^{n-1} \times \mathbb{C}^{2}$ has a product Ricci-flat Kähler cone metric as explained above.

(b) $n=4$. The unique minimizer is the weight $w_{*}=\left(1,1,1,1, \frac{2}{3}, \frac{2}{3}\right)$ with $\widehat{\operatorname{vol}}\left(w_{*}\right)=$ $\frac{50000}{27}$, degenerating $X$ to $A_{2}^{4} \times \mathbb{C}^{1}=\left\{z_{1}^{2}+\cdots+z_{4}^{2}+z_{6}^{3}=0\right\} \subset \mathbb{C}^{6}$. Notice that $A_{2}^{4} \times \mathbb{C}^{1}$ is semistable in the sense as explained above.

(c) $n=3$. The unique minimizer is the weight $w_{*}=\left(1,1,1, \frac{5}{9}, \frac{2}{3}\right)$, with $\widehat{\operatorname{vol}}\left(w_{*}\right)=$ $\frac{32000}{243}$, degenerating $X$ to $A_{2}^{3} \times \mathbb{C}^{1}=\left\{z_{1}^{2}+z_{2}^{2}+z_{3}^{2}+z_{5}^{3}=0\right\} \subset \mathbb{C}^{5}$. Notice that $A_{2}^{3} \times \mathbb{C}^{1}$ has a product Ricci-flat Kähler cone metric as explained above.

(d) $n=2$. The unique minimizer is the weight $w_{*}=\left(1,1, \frac{4}{9}, \frac{2}{3}\right)$ with $\widehat{\operatorname{vol}}\left(w_{*}\right)=$ $\frac{250}{27}$, coming from the natural $\mathbb{C}^{*}$-action.

(e) $n=1$. The unique minimizer is the weight $\left(1, \frac{4}{9}, \frac{2}{3}\right)$ with $\widehat{\operatorname{vol}}\left(w_{*}\right)=\frac{1}{12}$, coming form the natural $\mathbb{C}^{*}$-action.

3. Consider the $(n+1)$-dimensional $E_{6}$ singularity:

$$
X^{n+1}=E_{6}^{n+1}:=\left\{z_{1}^{2}+\cdots+z_{n}^{2}+z_{n+1}^{3}+z_{n+2}^{4}=0\right\} \subset \mathbb{C}^{n+2} .
$$

Consider the weights $\mathrm{x}$ such that $x_{1} \leq \cdots \leq x_{n}$. The results are:

(a) $n \geq 5$, the unique minimizer is $w_{*}=\left(1, \ldots, 1, \frac{n-2}{n-1}, \frac{n-2}{n-1}\right)$, degenerating $X$ to $A_{1}^{n-1} \times \mathbb{C}^{2}$.

(b) $n=4$, the unique minimizer is $\left(1,1,1,1, \frac{2}{3}, \frac{2}{3}\right)$, degenerating $X$ to $A_{2}^{4} \times \mathbb{C}^{1}=$ $\left\{z_{1}^{2}+\cdots+z_{4}^{2}+z_{5}^{3}=0\right\} \subset \mathbb{C}^{6}$.

(c) $n=3$, the unique minimizer is $\left(1,1,1, \frac{2}{3}, \frac{5}{9}\right)$, degenerating $X$ to $A_{2}^{3} \times \mathbb{C}^{1}=$ $\left\{z_{1}^{2}+z_{2}^{2}+z_{3}^{2}+z_{4}^{3}=0\right\} \subset \mathbb{C}^{5}$.

(d) $n=2$, the unique minimizer is $\left(1,1, \frac{2}{3}, \frac{1}{2}\right)$ with $\widehat{\text { vol }}=\frac{343}{36}$, coming from the natural $\mathbb{C}^{*}$-action.

(e) $n=1$, the unique minimizer is $\left(1, \frac{2}{3}, \frac{1}{2}\right)$ with $\widehat{\mathrm{vol}}=\frac{1}{6}$, coming from the natural $\mathbb{C}^{*}$-action.

4. Consider the $(n+1)$-dimensional $E_{8}$ singularity:

$$
X^{n+1}=E_{8}^{n+1}:=\left\{z_{1}^{2}+z_{2}^{2}+\cdots+z_{n}^{2}+z_{n+1}^{3}+z_{n+2}^{5}=0\right\} \subset \mathbb{C}^{n+2} .
$$

Consider the valuation determined by $\mathbf{x}=\left(x_{1}, \ldots, x_{n}, \alpha, \beta\right)$. Then

$$
A_{X}\left(v_{\mathbf{x}}\right)=\sum_{i=1}^{n} x_{i}+\alpha+\beta-\min \{2,3 \alpha, 5 \beta\}, \quad \operatorname{vol}\left(v_{\mathbf{x}}\right)=\frac{\min \left\{2 x_{1}, 3 \alpha, 5 \beta\right\}}{\left(\prod_{i=1}^{n} x_{i}\right) \alpha \beta} .
$$

(a) $n \geq 5$. $w_{*}=\left(1, \ldots, 1, \frac{n-2}{n-1}, \frac{n-2}{n-1}\right)$, degenerating $X$ to $A_{1}^{n-1} \times \mathbb{C}^{2} \subset \mathbb{C}^{n+2}$.

(b) $n=4$. $w_{*}=(1,1,1,1,2 / 3,2 / 3)$, degenerating $X$ to $A_{2}^{4} \times \mathbb{C}^{1}$.

(c) $n=3$. $w_{*}=(1,1,1,2 / 3,5 / 9)$, degenerating $X$ to $A_{2}^{3} \times \mathbb{C}^{1}$. 
(d) $n=2 . \quad w_{*}=(1,1,2 / 3,2 / 5)$ with $\widehat{\mathrm{vol}}=\frac{2048}{225}$, coming from the natural $\mathbb{C}^{*}$ action.

(e) $n=1 . w_{*}=(1,2 / 3,2 / 5)$ with $\widehat{\operatorname{vol}}=\frac{1}{30}$, coming from the natural $\mathbb{C}^{*}$-action.

Remark 8.1. In the $A-D-E$ type examples, notice that if $\operatorname{dim} X=2$ then $\widehat{\operatorname{vol}}\left(w_{*}\right)=\frac{4}{|G|}$, where $G$ is given by, $\mathbb{Z}_{k}$ for $A_{k-1}^{2}$, binary dihedral group of order $4(k-1)$ for $D_{k+1}$, and binary tetrahedral, octahedral, icosahedral groups for $E_{6}^{2}, E_{7}^{2}, E_{8}^{2}$ respectively. See [LL16] for a general result for quotient singularities.

\section{Appendix II: Second proof of Theorem 3.2}

In this appendix, we present a direct proof of Theorem 3.2 pointed out to me by an anonymous referee. This proof is more in the spiritual of [Izu85] and is based on the argument of Boucksom-Favre-Jonsson in [BFJ12, Section 3.1] and the following

Fact: (see [Betal09]) For any smooth projective variety $X^{\prime}$ and any ample line bundle $L \rightarrow X^{\prime}$, there exists a positive constant $\epsilon>0$ such that for any $x \in X^{\prime}$, the divisor $\pi^{*} L-\epsilon E$ is ample where $\pi$ is the blow-up at $x$ and $E$ is the exceptional divisor of $\pi$.

First, by compactifying $X^{\prime}$, we can assume $X^{\prime}$ is projective and $L$ is a very ample line bundle over $X^{\prime}$. Moreover we can assume $X^{\prime}$ is smooth and $\mu^{-1}(o)$ is a connected simple normal crossing divisor (not necessarily reduced) whose reduced support is given by $\sum_{i=1}^{m} F_{i}$. Indeed, the connectedness follows from Zariski's main theorem. Moreover we can take a log resolution of $\left(X^{\prime}, \mu^{-1}(o)\right)$ and the uniform estimate on the log resolution is easily seen to imply the uniform estimate for $\left(X^{\prime}, \mu^{-1}(o)\right)$. For any $o^{\prime} \in \mu^{-1}(o)$, let $\pi_{o^{\prime}}: B l_{o^{\prime}} X^{\prime} \rightarrow X^{\prime}$ be the blow-up of $o^{\prime}$ with the exceptional divisor denoted by $E_{0}$. By the above fact, we can choose $\epsilon$ sufficiently small so that $M=M_{o^{\prime}}=\pi_{o^{\prime}}^{*} L-\epsilon E_{0}$ is ample for any $o^{\prime} \in \mu^{-1}(o)$.

Remark 9.1. Although we don't need this, under the log-smoothness assumption, the dual complex $\Delta$ of $\mu^{-1}(o)$ is connected and the dual complex $\Delta_{o^{\prime}}$ of $\left(\pi_{o^{\prime}} \circ \mu\right)^{-1}(o)$ is obtained by either attaching a new segment at a vertex of $\Delta$ or taking a barycenter subdivision of a face of $\Delta$.

We will denote by $E_{i}$ the strict transform of $F_{i}$ under the blow up $\pi_{o^{\prime}}$. Assume $g \in \mathcal{O}_{X, o}$ and let $G=\left\{\mu^{*} g=0\right\}$ be the effective divisor. We write

$$
G=b_{0} E_{0}+\sum_{i=1}^{m} b_{i} E_{i}+\tilde{G}=\sum_{i=0}^{m} b_{i} E_{i}+\tilde{G}
$$

where $\tilde{G}$ is an effective divisor whose support does not contain any $E_{i}$. Notice that we have

$$
b_{i}=\operatorname{ord}_{F_{i}}\left(\mu^{*} g\right)=\operatorname{ord}_{F_{i}}(g), \text { for } 0 \leq i \leq m .
$$

In particular, we have $b_{0}=\operatorname{ord}_{E_{0}}(g)=\operatorname{ord}_{o^{\prime}}\left(\mu^{*} g\right)$. Next consider the intersection:

$$
\begin{aligned}
\sum_{j=0}^{m} b_{j}\left(E_{i} \cdot E_{j} \cdot M^{n-2}\right) & =\left(G \cdot E_{i} \cdot M^{n-2}\right)-\left(\tilde{G} \cdot E_{i} \cdot M^{n-2}\right) \\
& \leq\left(G \cdot E_{i} \cdot M^{n-2}\right)=0 .
\end{aligned}
$$

The last identity is because $G$ is a principle divisor near $E_{i}$. Set

$$
c_{i, j}:=\left(E_{i} \cdot E_{j} \cdot M^{n-2}\right) .
$$

Then from (32) we get the inequality:

$$
\sum_{j \neq i} b_{j} c_{i, j} \leq-b_{i} c_{i, i} \leq b_{i}\left|c_{i, i}\right|
$$


Without the loss of generality, we assume $E_{0} \cap E_{1} \neq \emptyset$. Then $c_{i, j} \geq 0$ if $j \neq i$ and

$$
\begin{aligned}
& c_{1,1}=\left(E_{1} \cdot E_{1} \cdot\left(\pi_{o^{\prime}}^{*} L-\epsilon E_{0}\right)^{n-2}\right)=\left(F_{1} \cdot F_{1} \cdot L^{n-2}\right)-\epsilon^{n-2}, \\
& c_{0,1}=\left(E_{0} \cdot E_{1} \cdot\left(\pi_{o^{\prime}}^{*} L-\epsilon E_{0}\right)^{n-2}\right)=\epsilon^{n-2}>0 .
\end{aligned}
$$

So we get $b_{0} \leq \frac{\left|c_{1,1}\right|}{c_{0,1}} b_{1}$ which is equivalent to:

$$
\operatorname{ord}_{o^{\prime}}\left(\mu^{*} g\right) \leq \frac{\left|c_{1,1}\right|}{c_{0,1}} \operatorname{ord}_{F_{1}}(g)
$$

By the original Izumi's theorem, we know that for each $i$ there exists a constant $d_{i}>0$ such that

$$
\operatorname{ord}_{F_{i}}\left(\mu^{*} g\right) \leq d_{i} \cdot \operatorname{ord}_{o}(g)
$$

So if we choose

$$
a_{2}=\max \left\{d_{i} \frac{\left|\left(F_{i} \cdot F_{i} \cdot L^{n-2}\right)-\epsilon^{n-2}\right|}{\epsilon^{n-2}}, 1 \leq i \leq m\right\},
$$

then we have the desired inequality:

$$
\operatorname{ord}_{o^{\prime}}\left(\mu^{*} g\right) \leq a_{2} \cdot \operatorname{ord}_{o}(g), \text { for any } g \in \mathcal{O}_{X, o} .
$$

\section{Acknowledgment}

The author is partially supported by NSF DMS-1405936. I would like to thank Yuchen Liu, Xiaowei Wang and Chenyang Xu for helpful discussions, and Gang Tian and Bernd Ulrich for their interest. I am grateful to Sebastien Boucksom and Mattias Jonsson for pointing out to me the existence of valuations with infinite log discrepancies. I also thank M. Jonsson for helping me realize the issue in Remark 4.5. I also would like to thank Yuji Odaka for helpful comment regarding the example 2.8. I am grateful to an anonymous referee for providing constructive suggestions on the presentation of paper and pointing out the second proof of Theorem 3.2 in Appendix II.

\section{References}

[AM69] M.F. Atiyah, and I.G. MacDonald, Introduction to commutative algebra, Addison-Wesley, Reading, Mass., 1969.

[Betal09] T. Bauer et al. A primer on Seshadri constants, Contemporary Mathematics, Volume 496, 2009.

[Blu16] H. Blum, Existence of valuations with smallest normalized volume, arXiv:1606.08894.

[Bor97] A. Borisov, Minimal discrepancies of toric singularities, Manuscripta Math. 92, 33-45 (1997).

[BFF12] S. Boucksom, T. de Fernex and C. Favre, The volume of an isolated singularity, Duke Math. J., Volume 161, Number 8 (2012), 1455-1520.

[BFFU13] S. Boucksom, T. de Fernex, C. Favre and S. Urbinati, Valuation spaces and multiplier ideals on singular varieties, arXiv:1307.02227.

[BFJ12] S. Boucksom, C. Favre and M. Jonsson, A refinement of Izumi's theorem, arXiv:1209.4104.

[BHJ15] S. Boucksom, T. Hisamoto, and M. Jonsson. Uniform K-stability, DuistermaatHeckman measures and singularities of pairs, arXiv:1504.06568. 
[Ber12] R. Berman, K-polystability of $\mathbb{Q}$-Fano varieties admitting Kähler-Einstein metrics, arXiv:1205.6214.

[CCT02] J. Cheeger, T. Colding and G. Tian, On the singularities of spaces with bounded Ricci curvature. Geom. Funct. Anal. 12 (2002), no. 5, 873-914.

[CDS15] X.X. Chen, S. K. Donaldson and S. Sun. Kähler-Einstein metrics on Fano manifols, I-III, J. Amer. Math. Soc. 28 (2015), 183-197, 199-234, 235-278.

[CS12] T. C. Collins, G. Székelyhidi, K-Semistability for irregular Sasakian manifolds, arXiv:1204.2230.

[Cut12] S.D. Cutkosky. Multiplicities associated to graded families of ideals. arXiv:1206.4077.

[dFH09] T. De Fernex and C. Hacon. Singularities on normal varieties, Compositio Math.145 (2009), 393-414.

[dFM15] de Fernex, and M. Mustaţă, The volume of a set of arcs on a variety. arXiv:1506.06424.

[Don02] S. Donaldson, Scalar curvature and stability of toric varieties, J. Differential Geometry. 62 (2002), no. 2, 289-349.

[DS15] S. Donaldson, S. Sun, Gromov-Hausdorff limits of Kahler manifolds and algebraic geometry, II, arXiv:1507.05082.

[Eis10] E. Eisenstein. Generalizations of the restriction theorem for multiplier ideals, arXiv:1001.2841.

[ELS01] L. Ein, R. Lazarsfeld, and K.E. Smith. Uniform bounds and symbolic powers on smooth varieties. Invent. Math. 144, 241-252 (2001).

[ELS03] L. Ein, R. Lazarsfeld, and K. E. Smith, Uniform approximation of Abhyankar valuation ideals in smooth function fields, American Journal of Mathematics 125 (2003), 409-440.

[FEM] T. de Fernex, L. Ein, M. Mustaţă, multiplicities and log canonical threshold, J. Algebraic Geometry.

[Fut83] A. Futaki, An obstruction to the existence of Einstein Kähler metrics, Inventiones Mathematicae (1983), 437-443.

[FOW09] A. Futaki, H. Ono, and G. Wang, Transverse Kähler geometry of Sasaki manifolds and toric Sasakian-Einstein manifolds, J. Differential Geometry, 83 (2009) 585-635.

[FJ04] C. Favre and M. Jonsson, The valuative tree. Lecture Notes in Mathematics, 1853, Springer-Verlag, Berlin, 2004

[Fuj15a] K. Fujita, On K-stability and the volume functions of $\mathbb{Q}$-Fano varieties, arXiv:1508.04052.

[Fuj15b] K. Fujita, Optimal bounds for the volumes of Kähler-Einstein Fano manifolds, arXiv:1508.04578.

[GMSY07] J. P. Gauntlett, D. Martelli, J. Sparks, S.-T. Yau, Obstructions to the existence of Sasaki-Einstein metrics, Commu. Math. Phy. 273, 803-827 (2007).

[HN] H-J. Hein, A. Naber. In preparation.

[Izu82] S. Izumi, Linear complementary inequalities for orders of germs of analytic functions, Invent. math. 65, 459-471 (1982).

[Izu85] S. Izumi. A measure of integrity for local analytic algebras. Publ. RIMS Kyoto Univ. 21 (1985), 719-735.

[Izu07] S. Izumi, Fundamental properties of germs of analytic mappings of analytic sets and related topics, Real and Complex Singularities, Proceedings of the Austrialian-Japanese Workshop, 2007 World Scientific Publishing Co. Pte. Ltd. 
[JM10] M. Jonsson, and M. Mustaţă, Valuations and asymptotic invariants for sequences of ideals, arXiv:1011.3699.

[KKL12] A.-S. Kaloghiros, A. Küronya and V. Lazić, Finite generation and geometry of models, arXiv:1202.1164.

[Kud01] S. A. Kudryavtsev, Pure log terminal blow-ups, Mathematical Notes, Volume 69, Issue 5, 814-819, 2001.

[Kur03] A. Küronya, A divisorial valuation with irrational volume, Journal of Algebra, 262 (2003) 413-423.

[Kol96] J. Kollár, Singularities of pairs, arXiv:9601026.

[Kol13] J. Kollár, Singularities of the Minimal Model Program, Cambridge Tracts in Mathematics, 200, Cambridge University Press, 2013.

[Laz96] R. Lazarsfeld, Positivity in algebraic geometry, I: Classical setting: line bundles and linear series, Erger Mathematik und ihrer Grenzgebiete. (3) 48, SpringerVerlag, Berlin, 1996.

[Li13] C. Li. Yau-Tian-Donaldson correspondence for K-semistable Fano varieties. To appear in Journal für die reine und angewandte Mathematik (Crelles journal), arXiv:1302.6681.

[Li15a] C. Li. Numerical solutions of Kähler-Einstein metrics on $\mathbb{P}^{2}$ with conical singularities along a smooth quadric curve. J. Geom. Anal., July 2015, vol. 25, issue $3,1773-1797$.

[Li15b] C. Li. K-semistability is equivariant volume minimization, arXiv:1512.07205.

[Liu16] Y. Liu, Upper bounds for the volumes of singular Kähler-Einstein Fano varieties, arXiv:1605.01034.

[LL16] C. Li, and Y. Liu, Kähler-Einstein metrics and volume minimization, arXiv:1602.05094.

[LS14] C. Li, S. Sun. Conical Kähler-Einstein metrics revisited. Comm. Math. Phys. 331 (2014), no. 3, 927-973.

[LWX14] C. Li, X. Wang and C. Xu; Degeneration of Fano Kähler-Einstein manifolds. arXiv:1411.0761.

[LX14] C. Li, C. Xu. Special test configurations and K-stability of Fano varieties, Ann. of Math. (2) 180 (2014), no.1, 197-232.

[LX16] C. Li, C. Xu. Stability of valuations and Kollár components, arXiv:1604.05398.

[Mar96] D. Markushevich, Minimal discrepancy for a terminal cDV singularity is 1, J. Math. Sci. Univ. Tokyo, 3 (1996), 445-456.

[MSY08] D. Martelli, J. Sparks, S.-T. Yau, Sasaki-Einstein manifolds and volume minimisation, Commu. Math. Phys. 280, 611-673 (2008).

[Mus02] M. Mustaţă, On multiplicities of graded sequences of ideals, Journal of Algebra 256 (2002) 229-249.

[MN12] M. Mustaţă, and J. Nicaise, Weight functions on non-archimedean analytic spaces and the Kontsevich-Soibelman skeleton, arXiv:1212.6328.

[Pro00] Yu. Prokhorov, Blow-ups of canonical singularities, Algebra (Moscow, 1998), de Gruyter, Berlin (2000), 301-317.

[Tak06] S. Takagi. Formulas for multiplier ideals on singular varieties. American Journal of Mathematics, 128 (2006), 1345-1362.

[Tia87] G. Tian, On Kähler-Einstein metrics on certain Kähler manifolds with $c_{1}(M)>$ 0, Invent. Math. 89, 225-246 (1987).

[Tia97] G. Tian, Kahler-Einstein metrics with positive scalar curvature, Invent. Math. 130 (1997), no. 1, 1-37. 
[Tia15] G. Tian, K-stability and Kähler-Einstein metrics, Communications on Pure and Applied Mathematics, Volume 8, Issue 7, pages 1085-1156, July 2015.

[Tou80] J.-Cl. Tougeron. Fonctions composées différentiables: Cas algébrique. Ann. Inst. Fourier (Grenoble) 30, 4, 51-74 (1980).

[Xu14] C. Xu, Finiteness of algebraic fundamental groups. Compos. Math. 150 (2014), Issue 03, 409-414.

Department of Mathematics, Purdue University, West Lafayette, IN 47907-2067 USA

li2285@purdue.edu 